\title{
Hans-Georg Becker \\ FRBR, Serials und CIDOC CRM - Modellierung von fortlaufenden Sammelwerken unter Verwendung von FRBRoo
}

\begin{abstract}
"The idea is [...] that FRBROO could be used as a formalism to transfer bibliographic data from the format in which it is produced to RDF, in order to be exposed as Linked Data. Catalogers are not expected to "produce FRBROO records," a phrase that has no meaning, anyhow. Rather, it is a matter of providing a framework for automated transformations from your local catalog to the Linked Data universe.”
\end{abstract}

Patrick Le Boeuf ${ }^{1}$

\section{Einleitung}

Die Modellierung fortlaufender Sammelwerke mittels des Referenzmodells Functional Requirements for Bibliographic Records (FRBR) gestaltet sich aus folgenden Gründen als schwierig. ${ }^{2}$

Unvollständigkeit fortlaufender Sammelwerke:

In den FRBR ist festgelegt, dass eine Manifestation in dem Sinn abgeschlossen sein muss, dass man ein Exemplar kaufen und ins Regal stellen kann. Bei fortlaufenden Sammelwerken ist dies nicht möglich, solange es weitere Hefte und Jahrgänge (im Fall von Zeitschriften) oder Bände (im Fall von Reihen oder Serien) gibt.

Heterogenität der in fortlaufenden Sammelwerken enthaltenen Manifestationen:

Teile einer Buchreihe können in einer weiteren Auflage, in einer anderen Reihe oder selbständig veröffentlicht werden; Zeitschriftenhefte können als Themenhefte wiederum als Buch veröffentlicht werden.

1 Vgl. Le Boeuf, Patrick: A Strange Model Named FRBROO. In: Cataloging \& Classification Quarterly: Routledge 2012. http://dx.doi.org/10.1080/01639374.2012.679222 (24.6.2012).

2 Vgl. auch Pohl, Adrian: Serials and FRBR. https://wiki1.hbz-nrw.de/display/SEM/2011/10/17/ Serials+and+FRBR (1.7.2012). 
Denkt man die Werke eher vom Inhalt und weniger von der physikalischen Erscheinungsform her, so lassen sich diese Probleme in der bibliographischen Beschreibung umgehen.

Der vorliegende Beitrag zeigt durch die Abbildung der Publikationstypen Einzelwerk und mehrbändig begrenztes Werk mittels FRBRoo eine Darstellung von fortlaufenden Sammelwerken auf, welche die genannten Probleme löst.

\section{Die CIDOC Ontologie für das kulturelle Erbe}

Beim CIDOC Conceptual Reference Model handelt sich um eine Norm (ISO 21127:2006) für den kontrollierten Austausch von Informationen im Bereich des kulturellen Erbes. Die Ontologie soll unter anderem von Archiven, Bibliotheken und Museen zur Verbesserung der Verfügbarkeit von Wissen angewandt werden. Es wurde vom CIDOC, einem der 30 internationalen Komitees des International Council of Museums (Internationalen Museumsrats, ICOM) entwickelt.

Mit dem CIDOC CRM wird das Ziel verfolgt, die vielfältigen Informationen im Bereich des kulturellen Erbes gemeinsam zu erfassen und einen allgemeinen Rahmen ihrer formalen Semantik zur Verfügung zu stellen, damit jede Information dieses Bereichs den Begriffen des CIDOC CRM zugeordnet werden kann. Auf diese Weise werden wichtige Voraussetzungen für die Informationsintegration geschaffen, da auf der Grundlage des CIDOC CRM Werkzeuge zur Schematransformation und -integration entwickelt werden können.

Das CRM beruht auf zwei Hierarchien von Entitäten und Eigenschaften und erlaubt ein hohes Maß an semantischer Präzision. Es eignet sich daher als eine Art Zwischenformat, dessen Verwendung die Anzahl der notwendigen Mappings dramatisch reduziert, wenn verschiedene Quellformate und mehrere Zielsprachen benötigt werden. ${ }^{3}$ Die wichtigste Eigenschaft des CIDOC CRM ist allerdings die Ereigniszentriertheit, d.h. es wird davon ausgegangen, dass jedes Objekt nur dann existiert, wenn vorher ein Ereignis stattgefunden hat, welches das Objekt zum Resultat hat.

3 Für eine Einführung in die Modellierung mittels Referenzontologien und Anwendungsontologien siehe auch Hohmann, Georg: Die Anwendung des CIDOC CRM für die semantische Wissensrepräsentation in den Kulturwissenschaften. In: Wissensspeicher in digitalen Räumen. Nachhaltigkeit, Verfügbarkeit, semantische Interoperabilität ; Proceedings der 11. Tagung der Deutschen Sektion der Internationalen Gesellschaft für Wissensorganisation, Konstanz, 20. bis 22. Februar 2008. Hrsg. von Jörn Sieglerschmidt. Würzburg: Ergon-Verl 2010. S. 211-222. 


\section{Mapping}

Das CRM erlaubt Mappings beliebiger Datenmodelle. Für einige der im bibliographischen Kontext einschlägigen Modelle liegen generische Mappings vor, die von den Entwicklern des CRM veröffentlicht wurden. Die im Rahmen des CRM-Entwicklungsprojekts entworfenen Mappings für Dublin Core (DC), Encoded Archival Description (EAD), Lightweight Information Describing Objects (LIDO) und andere Modelle und Formate orientieren sich an den im CRM-Entwicklungsprozess ausgearbeiteten Empfehlungen. ${ }^{4}$

Das Grundprinzip des CRM - und das macht das CRM als „Zwischensprache“ besonders interessant - besteht darin, die einzelnen in den Originalmetadaten vorgefundenen verschiedenen Entitäten, die ein Objekt ausmachen (z.B. bei einem Buch: der Autor, der Verlag), über entsprechende Events zu vermitteln. Konkret bedeutet das, dass zwischen Autor und Buch ein Schöpfungsereignis steht. Die Ereignisse ergeben in der Folge interessante Kandidaten für eine Deduplizierung von Objekten, da zwei identische Schöpfungsereignisse auf zwei identische Bücher verweisen. Formal werden dazu die Attribute zu Zeit- und Ortsangaben, sowie die durchführenden Akteure verglichen und bei Gleichheit die Ereignisse als äquivalent definiert. Die Ereignisse, die jeweils einen eindeutigen Identifier haben, bilden dadurch eine Gruppe, die selber auch wieder mit einem eindeutigen Identifier versehen wird. Diese Bündelung kann mit Hilfe der Bundle Ontology ebenfalls in den Linked Data Kontext übertragen und somit recherchierbar gemacht werden. ${ }^{5}$

Eine Konsequenz der Verwendung von Modellen wie dem CRM ist, dass die semantische Beschreibung der Objekte, die sich bei den Originalmetadaten aus dem bibliothekarischen Kontext mehr oder weniger stark an der Metapher des Bibliothekskatalogs bzw. des Karteikastens orientiert, in eine graphbasierte Darstellung übertragen wird.

\footnotetext{
4 ICOM: CIDOC CRM Mappings, Specializations and Data Examples. http://www.cidoc-crm.org/ crm_mappings.html (1.7.2012).

5 Bundle Ontology: http://purl.org/net/bundle.rdf (1.7.2012). Vgl. aber auch die Beschreibung im Wiki des Hochschulbibliothekszentrums NRW (hbz): Koreferenzen im Semantic Web. https:// wiki1.hbz-nrw.de/display/SEM/Koreferenzen+im+Semantic+Web\#KoreferenzenimSemanticW eb-bundle (1.7.2012).
} 


\section{FRBRoo: Harmonisierung der FRBR mit der Referenzontologie für kulturelles Erbe CIDOC CRM}

FRBRoo ist die objektorientierte Version der $F R B R^{6}$ und ermöglicht die gemeinsame Darstellung von Bibliotheks- und Museumsdokumentation. Damit ist es möglich, interoperable Informationssysteme für alle Nutzerinnen und Nutzer zu implementieren, die ein Interesse daran haben, auf gemeinsame oder verwandte Inhalte kultureller Einrichtungen zuzugreifen. ${ }^{7}$

Mit der Entwicklung der FRBRoo ging eine gegenseitige Anreicherung der FRBR und des CIDOC CRM einher:

- Ergänzung der FRBR um Zeit und Ereignisse,

- begriffliche Abklärung der Entität Manifestation,

- explizite Modellierung von Aufführungen und Aufzeichnungen, die in den FRBR erwähnt sind,

- Ergänzung des CRM durch die Entität Werk und

- Ergänzung des CRM durch einen Identifikator-Vergabeprozess.

FRBRoo fügt damit den FRBR die dynamischen Aspekte des CRM hinzu. Ferner erlaubt es, aufgrund der netzartigen Struktur des CRM, bibliographische Informationen in Linked Data Kontexte zu übertragen. Im Folgenden wird dies veranschaulicht.

Eine ausführliche Übersicht über verschiedene weitere Vokabulare zur Beschreibung bibliographischer Informationen in Linked Data Kontexten zeigt Carsten Klee in seinem Beitrag Vokabulare für bibliographische Daten in dem vorliegenden Band.

\section{Darstellung von Publikationstypen mittels FRBRoo}

In den folgenden Abschnitten werden Publikationstypen mit den FRBRoo dargestellt. ${ }^{8}$ Die Beschreibungen sind durch Diagramme unterstützt und mit entspre-

6 Das „oo“ in FRBRoo steht für „object-oriented“.

7 Vgl. Riva, Pat, Martin Doerr u. Maja Žumer: FRBRoo: eine Basis zur gemeinsamen Informationsdarstellung in Kulturerbe-Institutionen. http://ifla.queenslibrary.org/IV/ifla74/papers/156Riva_Doerr_Zumer-trans-de.pdf (1.7.2012).

8 Eine erste Version dieser Abbildung wurde veröffentlicht in Becker, Hans-Georg: MODS2FRBROO. Ein Tool zur Anbindung von bibliografischen Daten an eine Ontologie für Begriffe und Informationen im Bereich des kulturellen Erbes. In: Semantic Web \& Linked Data. Elemente zukünftiger Informationsinfrastrukturen ; 1. DGI-Konferenz ; 62. Jahrestagung der DGI ; Frankfurt am Main, 7. - 9. Oktober 2010 ; Proceedings. Hrsg. von Marlies Ockenfeld. Frankfurt am Main: 
chenden Belegen aus der Spezifikation der FRBRoo und dem CRM angereichert. Die Modellierung stützt sich vor allem auf die Ereigniszentriertheit des CRM, wodurch die Schöpfungs-, Publikations- und Produktionsprozesse transparenter werden.

\section{Publikationstypen nach Gantert/Hacker}

Der Betrachtung der Publikationstypen werden die Definitionen im bibliothekarischen Grundlagenbuch von Gantert/Hacker (Gantert, 2008) zugrunde gelegt. Zur Verdeutlichung der hier betrachteten Aspekte der Publikationstypen, werden diese im Folgenden nochmals aufgeführt.

- Einzelwerk

Ein Einzelwerk ist eine in sich geistige abgeschlossene Schöpfung, die zur zusammenhängenden Veröffentlichung vorgesehen ist und in einem oder mehreren Teilen erscheint.

- Sammlung

Als Sammlung wird eine Veröffentlichung bezeichnet, in der zwei oder mehr Einzelwerke desselben Verfassers vereinigt sind.

- Sammelwerk

Unter Sammelwerk versteht man ein Buch mit mindestens zwei Einzelwerken von zwei oder mehr Verfassern.

- Fortsetzungswerk

Fortsetzungswerk nennt man eine mehrbändige Publikation, bei der die einzelnen Bände oder Teile nacheinander in zeitlichen Abständen erscheinen. Der Unterschied zur Serie liegt darin, dass Fortsetzungswerke nach einer bestimmten Anzahl von Bänden oder Teilen abgeschlossen sind.

Die zentrale Rolle beim Mapping mit den FRBRoo stellt die Verfeinerung des Werk-Begriffs der FRBR in den FRBRoo dar.

Die Ausführungen in den originalen FRBR zu den Gruppe-1-Entitäten lassen in einigen Fällen verschiedene Interpretationen $\mathrm{zu}$, die in Teilen $\mathrm{zu}$ logischen Inkonsistenzen führen. Insbesondere die Entität Work deckt mehrere Szenarien ab, die in der Realität verschiedene Eigenschaften haben. ${ }^{9}$ Die FRBRoo greifen diese Ungenauigkeit auf und definieren das Work als Superklasse und verfeinern diese dann durch Work-Begriffe, welche die verschiedenen Szenarien modellieren können.

DGI Dt. Ges. für Informationswiss. und Informationspraxis 2010. S. 77-86.

9 Im Folgenden werden die Entitäten und Eigenschaften von CRM und FRBRoo mit ihren englischen Originalbezeichnungen verwendet. 


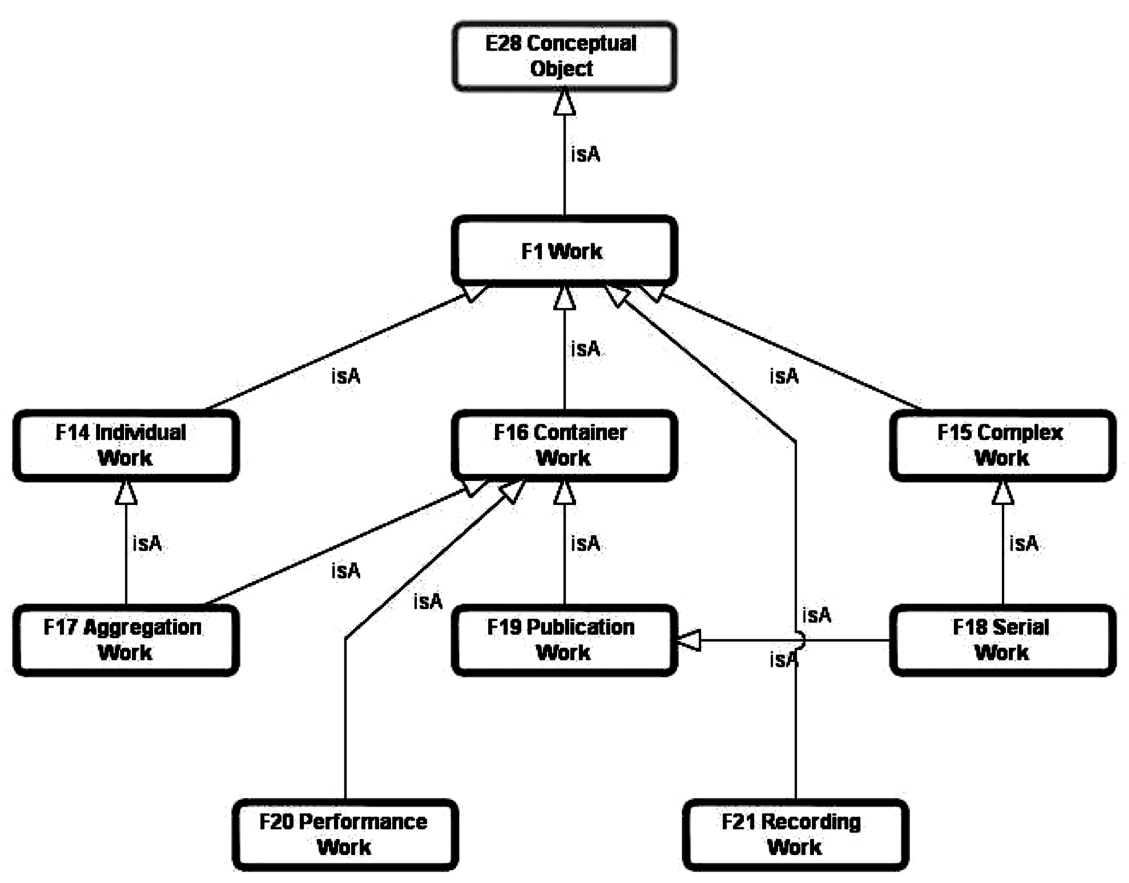

Abbildung 1: Die Klassenhierarchie zur FRBR-Entität Work.

So entsteht beispielsweise eine Entität Serial Work, die zum einen den intellektuellen Anteil des Verlags berücksichtigt (Publication Work) und zum anderen eine Umgebung für die Aggregation anderer Werke bildet (Complex Work). Dabei ist die soeben genannte Aggregation nicht mit der Aggregation bei Sammelwerken zu verwechseln. Letztere findet auf der Ebene der Expressions statt. In den FRBRoo heißt es zu Aggregation Work:

This class comprises works whose essence is the selection and/or arrangement of expressions of one or more other works. This does not make the contents of the aggregated expressions part of this work, but only part of the resulting expression. F17 Aggregation Work may include additional original parts.

Somit fallen hierunter die Sammelwerke und Sammlungen.

Legt man nun die Publikationstypen nach dem bibliothekarischen Standardwerk von Gantert/Hacker zugrunde, so ergibt sich für die Publikationstypen das Bild in Abbildung 2. Die Abbildung enthält zwei Typen von Relationen: Unter-/ Oberklassen (subClassOf) und Teil/Ganzes-Beziehungen (hasPart). Letztere 
stellen dabei - die insbesondere im deutschen Bibliothekswesen starken - Verknüpfungen zu Überordnungen dar.

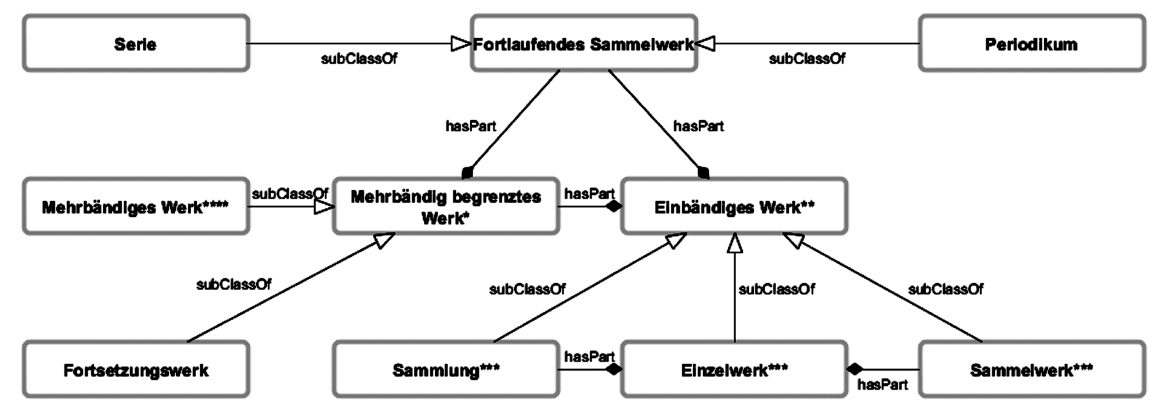

Abbildung 2: Publikationstypen nach Gantert/Hacker.

Für mehrbändig begrenzte Werke im Sinne von bibliographischen Einheiten (gekennzeichnet durch *) werden zwei Fälle unterschieden: mehrbändig begrenzt mit zeitgleich erschienenen Bänden (gekennzeichnet durch $\star \star \star \star)$ und Fortsetzungswerke. Ein einbändiges Werk im Sinne einer bibliographischen Einheit (gekennzeichnet durch ${ }^{\star \star}$ ) kann ebenfalls verschiedene Formen annehmen, beispielsweise als Sammlung oder Sammelwerk.

Abbildung 3 stellt nun die Zuordnung $\mathrm{zu}$ den FRBRoo-Work-Entitäten (sameAs) dar. Ferner kann der Abbildung entnommen werden, dass sich Publikationstypen FRBRoo-Klassen „teilen“. Am Beispiel des fortlaufenden Sammelwerks sind dies die Serie/Reihe und das Periodikum, welche sich das Serial Work teilen. Der formale Unterschied zwischen Serien und Periodika ist „nur“ die Beschreibung der Erscheinungsweise. Diese kann aber in einem Modell, wie es die FRBRoo sind, durch die Verwendung entsprechender Vokabulare für die Attribute der Entitäten beschrieben werden.

In den folgenden Abschnitten werden nun die einzelnen Publikationstypen mittels FRBRoo dargestellt, die für die Herleitung eines Modells für fortlaufende Sammelwerke notwendig sind. Begonnen wird mit der Abbildung von Einzelwerken.
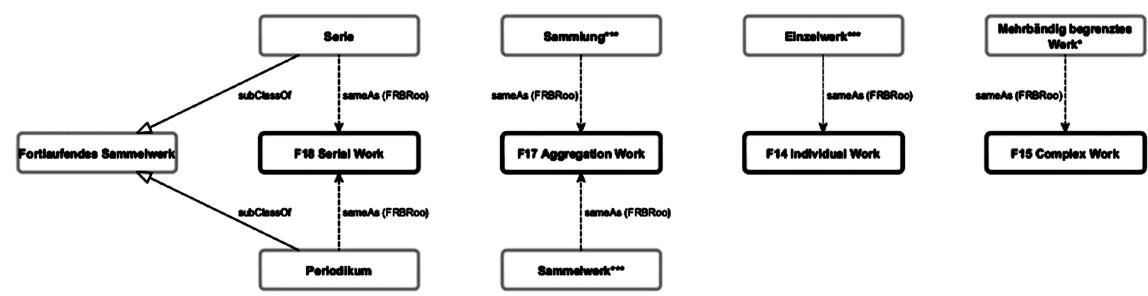

Abbildung 3: Zuordnung der FRBRoo-Work-Entitäten zu den Publikationstypen. 


\section{Einzelwerke}

Das CRM und somit die FRBRoo liefern durch ihre Ereigniszentriertheit für das Modell drei wesentliche Prozesse: den Schöpfungsprozess (realisiert durch das Ereignis F28 Expression Creation), ${ }^{10}$ den Publikationsprozess (realisiert durch das Ereignis F30 Publication Event) ${ }^{11}$ und den Produktionsprozess (realisiert durch das Ereignis F32 Carrier Production Event). ${ }^{12}$

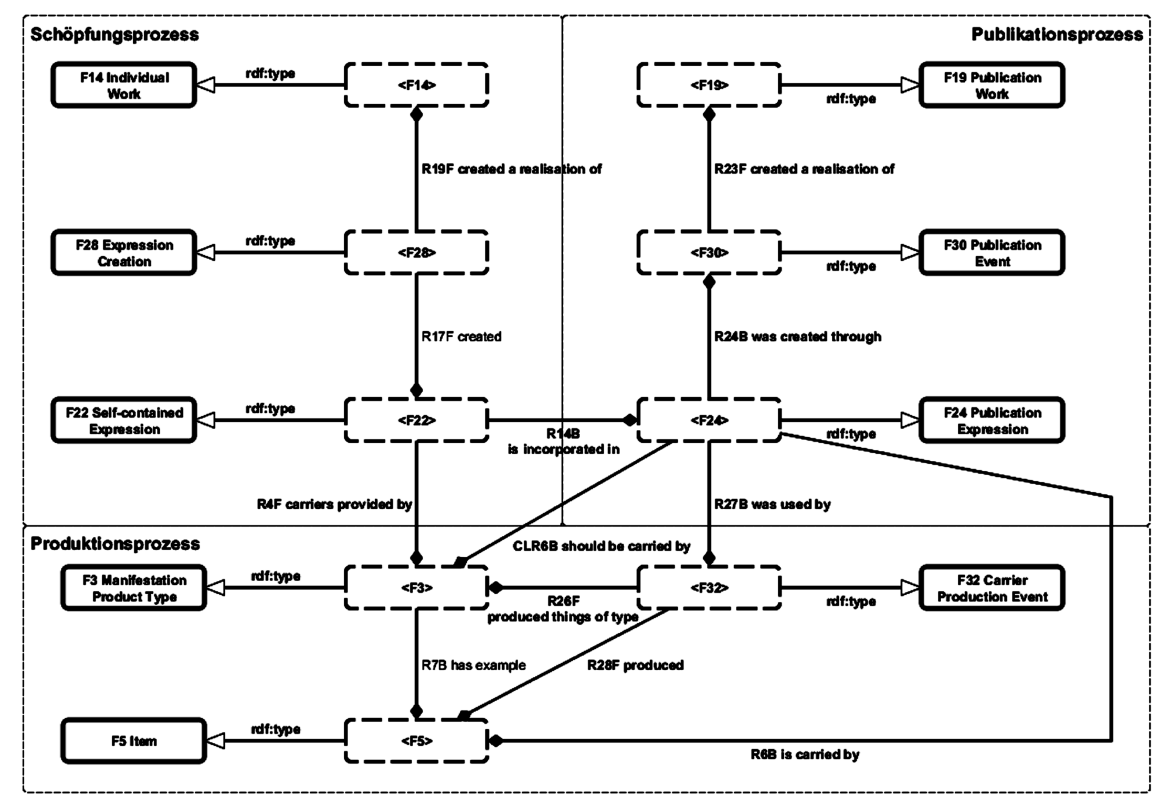

Abbildung 4: Abbildung eines Einzelwerks.

Die Beschreibung des Publikationstyps Einzelwerk stellt in einem gewissen Sinne ein Kernmodell dar. So werden darin die grundsätzliche Unterscheidung und die Zusammengehörigkeit der oben genannten Prozesse sichtbar. Die in diesen Prozessen verwendeten Entitäten und Relationen werden bei sämtlichen Publikationstypen wieder verwendet und den Gegebenheiten angepasst. Der Übersichtlichkeit halber wird das Modell in zwei Teilen präsentiert: dem Modell für die

10 Vgl Bekiari, Chryssoula, Martin Doerr u. Patrick Le Bœuf: Definition of Object-Oriented FRBR. http://www.cidoc-crm.org/docs/frbr_oo/frbr_docs/FRBRoo_V1.0.2.pdf (1.7.2012) S. 14, Abb. 1.

11 Vgl. ebd. S. 15, Abb. 2.

12 Vgl. ebd. S. 18, Abb. 5. 
materiellen Konzepte und die Ereignisse (Abbildung 4), sowie dem Modell der Akteure (Abbildung 5). ${ }^{13}$

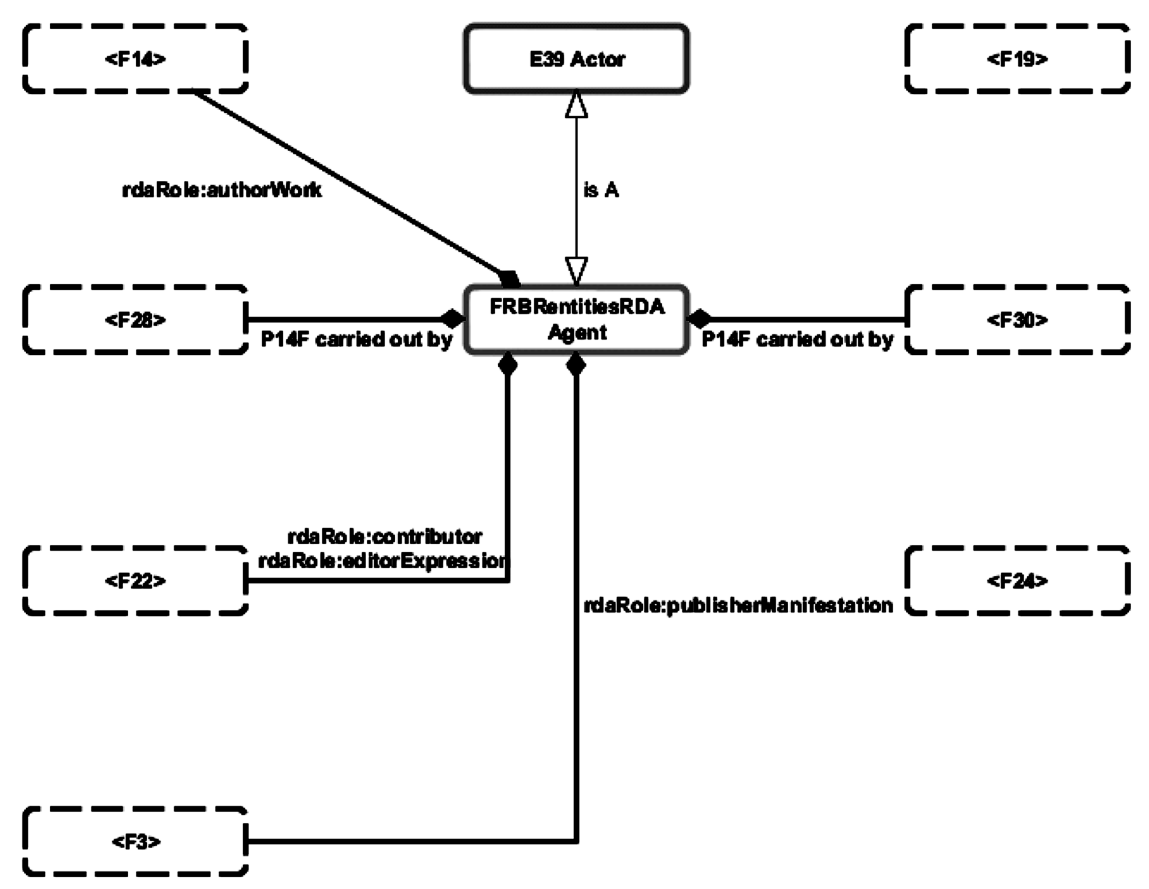

Abbildung 5: Modellierung der Akteure zu den Ereignissen.

Die Linien der Schöpfungs- und Publikationsprozesse verbinden sich auf der Ebene der Expressions. Hier wird der Tatsache Rechnung getragen, dass der Verlag durch das Hinzufügen seines Layouts und sonstiger, ergänzender Teile die Expression und somit die Realisierung des Schöpfers anreichert. Diese erweiterte Expression wird dann in den Produktionsprozess weitergeleitet. ${ }^{14}$

Aus der Sicht eines Bibliothekskatalogs sind in der Regel zum Schöpfungsprozess des Werks wenige bis keine Informationen bekannt. Die formalen Beschreibungen der Werksebene werden im Bibliothekskontext entsprechend auf der Seite des Publikationsprozesses abgelegt, d.h. insbesondere, dass der Titel, so wie er bei der formalen Erschließung erfasst wird, als Attribut zum F19 Publication Work gehört. Anders ist das bei der Zuordnung der Akteure des Schöpfungsprozesses.

13 Die Abbildungen enthalten grundsätzlich zwei Knotentypen: die mit der durchgehenden Umrandung sind die Klassen der Ontologie, die mit der gestrichelten Umrandung sind Instanzen. 14 Hier ist zu beachten, dass die Relation R4 in der Abbildung eine Abkürzung der Relationskette F22 R14B F24 CLR6B F3 darstellt. 
Hier ist bei der formalen Erschließung bekannt, dass die angegebenen Personen oder Körperschaften an der Realisierung der Expression mitgewirkt haben.

Die Akteure werden in dem vorliegenden Modell zum einen als Ausführende direkt an die Ereignisse angebunden (unter Verwendung der Relation P14 carried out by des CRM), zum anderen werden die Rollen der Akteure mithilfe des $R D A$ Vokabulars modelliert. ${ }^{15,16}$

Die Abbildung 5 zeigt die Verwendung eines weiteren Modells, den FRBRentitesRDA. ${ }^{17}$ Hierbei handelt es sich um die Definition der FRBR-Entitäten aus dem $R D A$-Modell. An dieser Stelle soll nun die Verbindung der Modelle genauer aufgezeigt werden.

Die FRBRoo greifen, wie oben erwähnt, die Schwachstellen der FRBR auf und versuchen diese zu beheben. Dabei verlassen sie aber nie das Grundvokabular der FRBR-Entitäten. So bleiben die Entitäten Work, Expression, Manifestation und Item in FRBRoo erhalten und bekommen nur die CRM-typische Nummer verliehen. Auch die $R D A$ basieren auf den Konzepten der FRBR. Somit sind die vier Entitäten der FRBR-Gruppe 1 in allen drei Modellen äquivalent bzw. im Fall von F3 Manifestation-Product Type und F4 Manifestation Singleton in FRBRoo abgeleitet. ${ }^{18}$ Diese Feststellung ist insofern wichtig, dass es nur dann möglich ist,

15 RDA - Resource, Description and Access; das neue Regelwerk für die Erschließung von kulturellen Objekten. Das CRM liefert selber auch eine Möglichkeit, den Ausführenden eines Ereignisses eine Rolle zuzuordnen. Und zwar sieht das CRM eine Relation P14.1 in the role of der Relation P14 carried out by vor. Dieses Konstrukt ist in dieser Form aber nicht RDF-konform und müsste durch die Modellierung eines sogenannten Blank-Nodes aufgelöst werden. Da die Verwendung von Blank-Nodes in der Praxis auch nicht unbedingt empfohlen wird (vgl. die folgende Fußnote) und das Bibliothekswesen mit den $R D A$ eine geeignete Alternative hat, wird diese hier auch verwendet. Vor der gleichen Herausforderung stand man auch in Mazurek, Cezary, Krysztof Sielski u. a.: From MARC21 and Dublin Core, through CIDOC CRM. First Tenuous Steps towards Representing Library Data in FRBRoo. Accepted Paper for the CIDOC Conference 2012, Helsinki. http://www.cidoc2012.fi/en/File/1611/mazurek.pdf (1.7.2012). Allerdings hat man dort den Weg der Verfeinerung der F28 Expression Creation eingeschlagen.

16 Vgl. z.B. Heath, Tom u. Christian Bizer: Linked Data. Evolving the web into a global data space. 1. Aufl. San Rafael, Calif: Morgan\& Claypool 2011 (=Synthesis lectures on the semantic web theory and technology 1). 2.4.1, Absatz "RDF Features Best Avoided in the Linked Data Context": "The scope of blank nodes is limited to the document in which they appear, meaning it is not possible to create RDF links to them from external documents, reducing the potential for interlinking between different Linked Data sources. In addition, it becomes much more difficult to merge data from different sources when blank nodes are used, as there is no URI to serve as a common key. Therefore, all resources in a data set should be named using URI references.”

17 FRBRentitiesRDA: http://rdavocab.info/uri/schema/FRBRentitiesRDA (13.10.2012)

18 Im Fall der Manifestations ist ein direktes Mapping aufgrund der Aufteilung in F3 und F4 in den FRBRoo nicht möglich. Hier werden stattdessen die Instanzen einer Manifestation über rdf:type zum einen F3 bzw. F4 und zusätzlich der FRBRentitiesRDA:Manifestation zugeordnet. 
Relationen und Attribute aus verschiedenen Modellen und Ontologien zu benutzen, wenn die Entitäten in der beschriebenen Weise zusammenpassen. Nur unter diesen Voraussetzungen kann die Idee des Linked Data funktionieren.

Im vorliegenden Beitrag werden die Entitäten der FRBRoo mit denen der FRBRentitiesRDA „gematcht“, um nicht sämtliche Metadaten zu den Instanzen als CRM oder FRBRoo-Tripel zu beschreiben, was den Graph in seiner Größe förmlich explodieren ließe. ${ }^{19}$ Der Vorteil hierbei ist, dass ab einer bestimmten Tiefe des Graphen nur noch bereits bekannte und hoffentlich standardisierte Metadaten verwendet werden.

Tabelle 1: Ontologie-Mappings

\begin{tabular}{lll}
\hline Instanz & rdf:type & Mapping zu anderer Ontologie \\
\hline Work & F1 Work & rdfs:subClassOf FRBRentitiesRDA:Work \\
Expression & F2 Expression & rdfs:subClassOf FRBRentitiesRDA:Expression \\
Item & F5 Item & rdfs:subClassOf FRBRentitiesRDA:Item \\
Actor & E39 Actor & owl:sameAs FRBRentitiesRDA:Agent \\
Person & F10 Person & owl:sameAs FRBRentitiesRDA:Person \\
& & owl:sameAs E21 Person \\
Corporate Body & F11 Corporate Body & owl:sameAs FRBRentitiesRDA:CorporateBody \\
& & rdfs:subClassOf E74 Group \\
\hline
\end{tabular}

Unter der Annahme der Matchings in Tabelle 1 können für die in diesem Beitrag verwendeten Konzepte die Data Properties des RDA-Modells verwendet werden. Diese werden seit einigen Jahren, obwohl das Regelwerk noch nicht verabschiedet ist, bereits in $R D F$ modelliert und im Netz bereitgestellt. ${ }^{20}$ Die Relationen in Abbildung 5 verwenden ohnehin bereits das Vokabular RDA Roles aus dieser Sammlung. ${ }^{21}$

Für den Schöpfungsprozess finden die Rollen authorWork, contributor und editorExpression, und für den Publikationsprozess die Rolle publisherManifes-

19 Verwendet man nur das CRM, so müssen für Aussagen sehr viele Tripel erzeugt werden, bis die Semantik klar ausgedrückt wird, wodurch sehr große Graphen entstehen. Um diese Graphen kleiner zu halten, wird mittels Ontology Alignment ein Mapping zu Modellen erzeugt, die für die gleiche Aussagekraft kürzere Wege erlauben. So entsteht ein durch das CRM zusammengehaltener Graph, dessen Knoten durch fachspezifische Ontologien beschreiben werden. Auch Mazurek, Cezary u. Sielski, Krysztof u. a.: MARC21 hatten das Problem. Sie zeigen in ihrem Beitrag an einem Beispiel, wie kleinteilig diese Art der Modellierung wäre.

20 The Registry!: The RDA (Resource Description and Access) Vocabularies. http://rdvocab.info/ (1.7.2012).

21 RDA roles: http://rdavocab.info/roles (13.10.2012) 
tation Verwendung und werden über den Akteur dem entsprechenden Ereignis zugeordnet.

Das bisher beschriebene Modell wird im Folgenden durch ein Beispiel verdeutlicht. Dabei wird auch für weitere Attribute das $R D A$-Vokabular verwendet. ${ }^{22}$

\section{Beispiel 1:}

FRBR : a guide for the perplexed / Maxwell, Robert L. Chicago: American Library Association, 2008. VII, 151 S. : graph. Darst.

ISBN: 978-0-8389-0950-8, 0-8389-0950-7

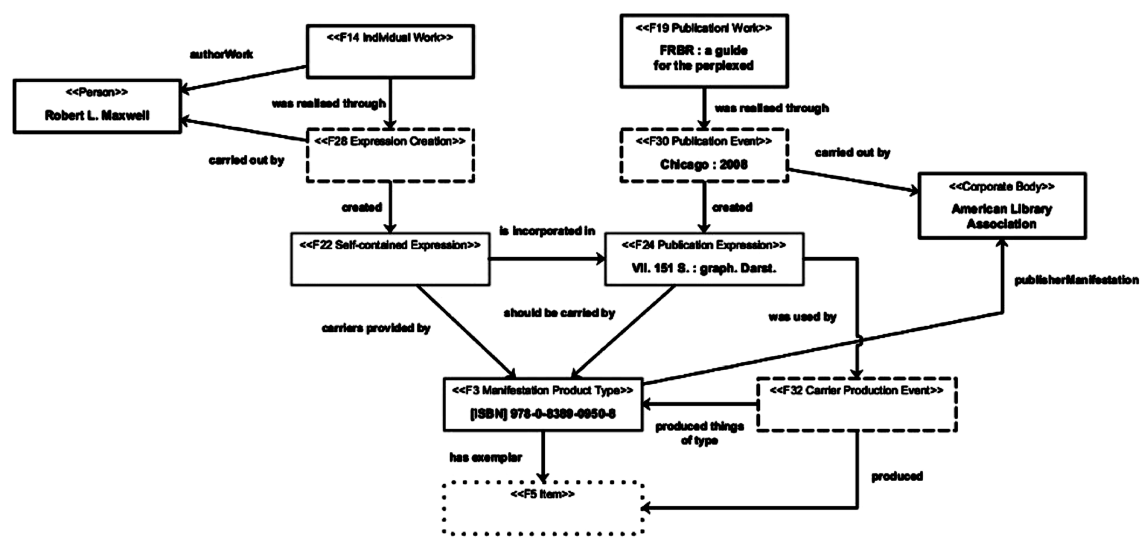

Abbildung 6: Graphische Darstellung von Beispiel 1.

Wir gehen nun ebenenweise durch die FRBRoo-Entitäten. Die Ebene der Exemplare wird dabei vernachlässigt. Die Darstellung erfolgt in validem Turtle und nutzt folgende Präfixe:

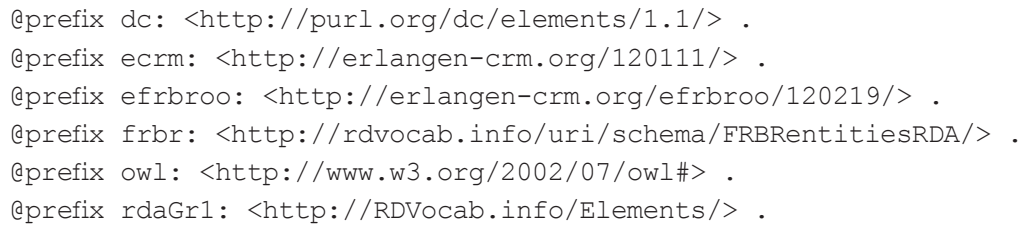

22 Die Links führen zum Triple Store der Linked Open Library Data-Plattform der Universitätsbibliothek Dortmund (Universitätsbibliothek Dortmund: Offene bibliographische Daten an der Universitätsbibliothek Dortmund. http://data.ub.tu-dortmund.de/projekte/offene-daten/ (13.10.2012)) bzw. zum Triple Store der bibliographischen Daten aus dem DFG-Projekt ArcheoInf (ArcheoInf Dokumentenrepositorium. http://archeoinf.ub.rub.de/ (1.7.2012)). 
Aprefix rdaRole: <http://RDVocab.info/roles/> .

eprefix rdf: <http://www.w3.org/1999/02/22-rdf-syntax-ns\#> .

eprefix gnd: <http://d-nb.info/gnd/> .

eprefix marcLang: <http://marccodes.heroku.com/languages/> .

eprefix lobid: <http://lobid.org/resource/> .

eprefix f14: <http://data.ub.tu-dortmund.de/resource/ubdo/frbroo/F14/> .

@prefix f19: <http://data.ub.tu-dortmund.de/resource/ubdo/frbroo/F19/> .

eprefix f28: <http://data.ub.tu-dortmund.de/resource/ubdo/frbroo/F28/> .

eprefix f30: <http://data.ub.tu-dortmund.de/resource/ubdo/frbroo/F30/> .

eprefix f22: <http://data.ub.tu-dortmund.de/resource/ubdo/frbroo/F22/> .

eprefix f24: <http://data.ub.tu-dortmund.de/resource/ubdo/frbroo/F24/> .

eprefix fll: <http://data.ub.tu-dortmund.de/resource/ubdo/frbroo/F11/> .

eprefix f3: <http://data.ub.tu-dortmund.de/resource/ubdo/frbroo/F3/> .

eprefix f32: <http://data.ub.tu-dortmund.de/resource/ubdo/frbroo/F32/> .

Individual Work:

f14:HT015671602

efrbroo:R19i_was_realised_through f28:HT015671602 ;

rdaRole:authorWork gnd:142840386;

rdf:type efrbroo:F14_Individual_Work .

Publication Work:

f19:HT015671602

efrbroo:R23i_was_realised_through $\mathrm{f} 30$ :HT015671602 ;

rdaGr1:preferredTitleForTheWork FRBR : a guide for the perplexed ;

rdf:type efrbroo:F19_Publication_Work .

Expression Creation:

f28:HT015671602

ecrm:P14_carried_out_by gnd:142840386 ;

efrbroo:R17_created f22:HT015671602 ;

efrbroo:R19_created_a_realisation_of f14:HT015671602 ;

rdf:type efrbroo:F28_Expression_Creation .

Publication Event:

f30:HT015671602

ecrm:P14_carried_out_by f11:HT015671602-1 ;

dc:date 2008 ;

dc:place Chicago ;

efrbroo:R23_created_a_realisation_of f19:HT015671602 ;

efrbroo:R24_created f24:HT015671602 ;

rdf:type efrbroo:F30_Publication_Event . 
Self-Contained Expression:

f22:HT015671602

efrbroo:R14i_is_incorporated_in f24:HT015671602 ;

efrbroo:R17i_was_created_by f28:HT015671602 ;

efrbroo:R4_carriers_provided_by f3:HT015671602 ;

rdaGr1:languageOfTheContentExpression marcLang:eng ;

rdf:type efrbroo:F22_Self-Contained_Expression .

Publication Expression:

f24:HT015671602

efrbroo:CLR6i_should_be_carried_by f3:HT015671602 ;

efrbroo:R14_incorporates f22:HT015671602 ;

efrbroo:R24i_was_created_through f30:HT015671602 ;

efrbroo:R27i_was_used_by f32:HT015671602 ;

rdf:type efrbroo:F24_Publication_Expression .

Manifestation:

f3:HT015671602

efrbroo:CLR6_should_carry f24:HT015671602 ;

efrbroo:R26i_was_produced_by f32:HT015671602 ;

efrbroo:R4i_comprises_carriers_of f22:HT015671602 ;

owl:sameAs lobid:HT015671602 ;

rdaGr1:dateOfPublicationManifestation 2008;

rdaGr1:identifierForTheManifestation [ISBN] 0-8389-0950-7 ;

rdaGr1:identifierForTheManifestation [ISBN] 978-0-8389-0950-8 ;

rdaGr1:placeOfPublicationManifestation Chicago ;

rdaGr1:placeOfPublicationManifestation XD-US ;

rdaGr1:publicationStatementManifestation

Chicago : American Library Association, 2008

rdaGr1:publishersNameManifestation American Library Association ;

rdaRole:publisherManifestation f11:HT015671602-1 ;

rdf:type efrbroo:F3_Manifestation_Product_Type ;

rdf:type frbr:Manifestation .

\section{Sammelwerke und Sammlungen}

Ein Sammelwerk oder eine Sammlung zeichnet sich dadurch aus, dass es Expressions anderer Werke in sich vereint oder anders ausgedrückt aggregiert. Für diese Werke gibt es in den FRBRoo die Entität F17 Aggregation Work, welches die Eigenschaften des F14 Individual Work und des F16 Container Work in sich vereint: 
This class comprises works whose essence is the selection and/or arrangement of expressions of one or more other works. This does not make the contents of the aggregated expressions part of this work, but only part of the resulting expression. F17 Aggregation Work may include additional original parts. ${ }^{23}$

An diesem Publikationstyp sind somit mehrere Werke und deren Realisierungen beteiligt: zum einen sind dies die Werke der Beiträge, zum anderen ist es das Sammelwerk an sich. Daraus folgt auch, dass es einige zu betrachtende Prozesse gibt.

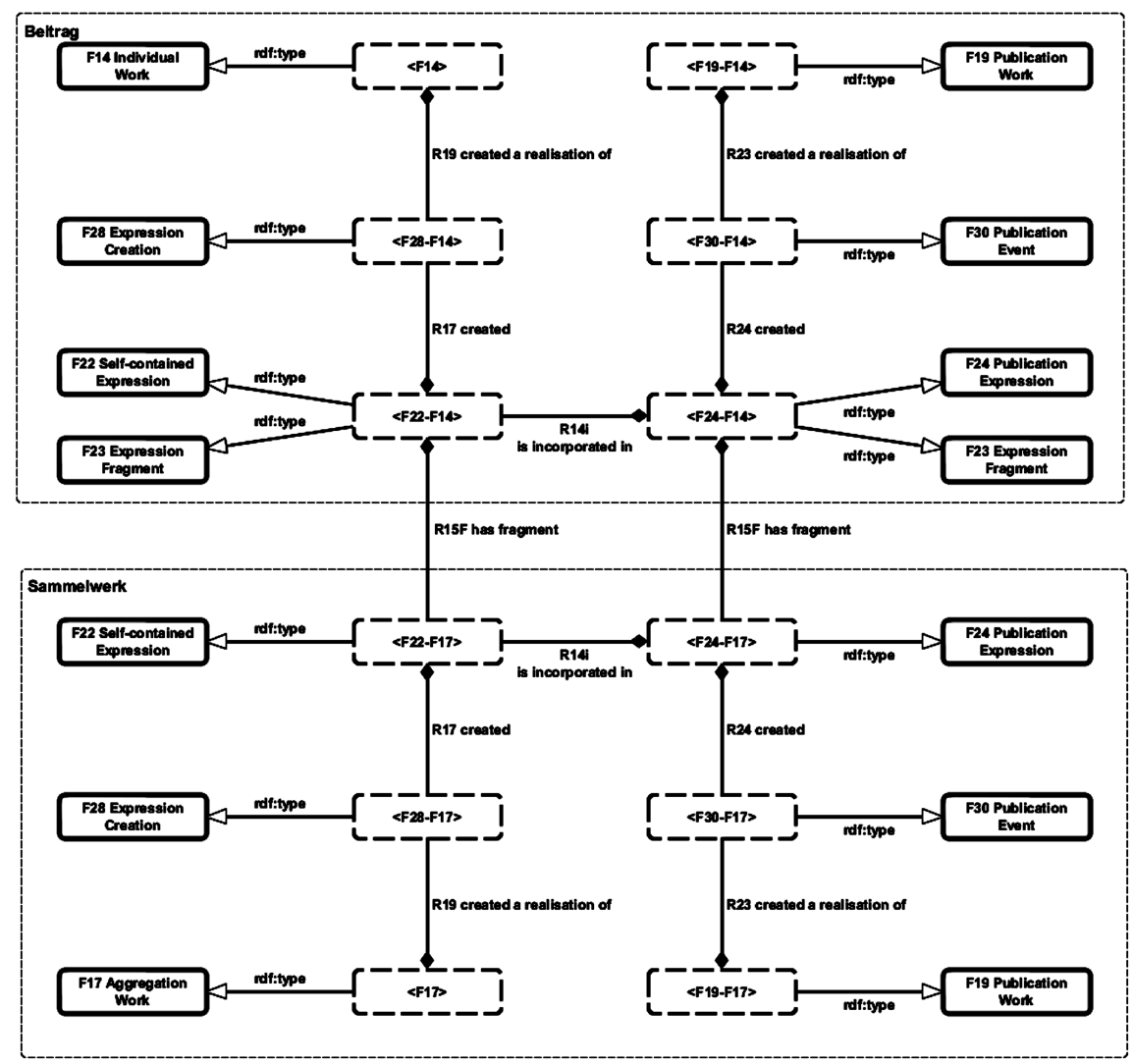

Abbildung 7: Schöpfungs- und Publikationsprozess eines Sammelwerkes.

Die Schöpfungsprozesse sind zunächst mal dem des Einzelwerks sehr ähnlich (bei den Beiträgen handelt es sich um Einzelwerke, beim Sammelwerk handelt es sich um ein F17 Aggregation Work, welches von F14 Individual Work abgeleitet ist).

23 Vgl. Bekiari, Chryssoula, Martin Doerr u. Patrick Le Bœuf: Definition. S. 46. 
Allerdings kommt hinzu, dass die aus den Beiträgen entstandenen Expressions neben ihrer Eigenschaft sich selbst zu enthalten (F22 Self-contained Expression) auch Fragmente (F23 Expression Fragment) einer übergeordneten Expression, der des Sammelwerks, sind. Abbildung 7 stellt dies graphisch dar. Somit ist eine Expression eines Beitrags gleichzeitig Instanz von F22 und F23.

Die soeben beschriebene Fragmentierung gilt auch im Publikationsprozess. So erhält jede Expression eines Beitrags eine eigene F24 Publication Expression. Somit gilt für die Expressions im Publikationsprozess, dass sie gleichzeitig Instanzen von F24 und F23 sind.

\section{Beispiel 2:}

Commercial Interchanges Between Greeks and Natives / Graham, Alexander JohnIn: Collected papers on Greek colonization / Graham, Alexander John (Hrsg.) Leiden, Boston, Köln : Brill, 2001.

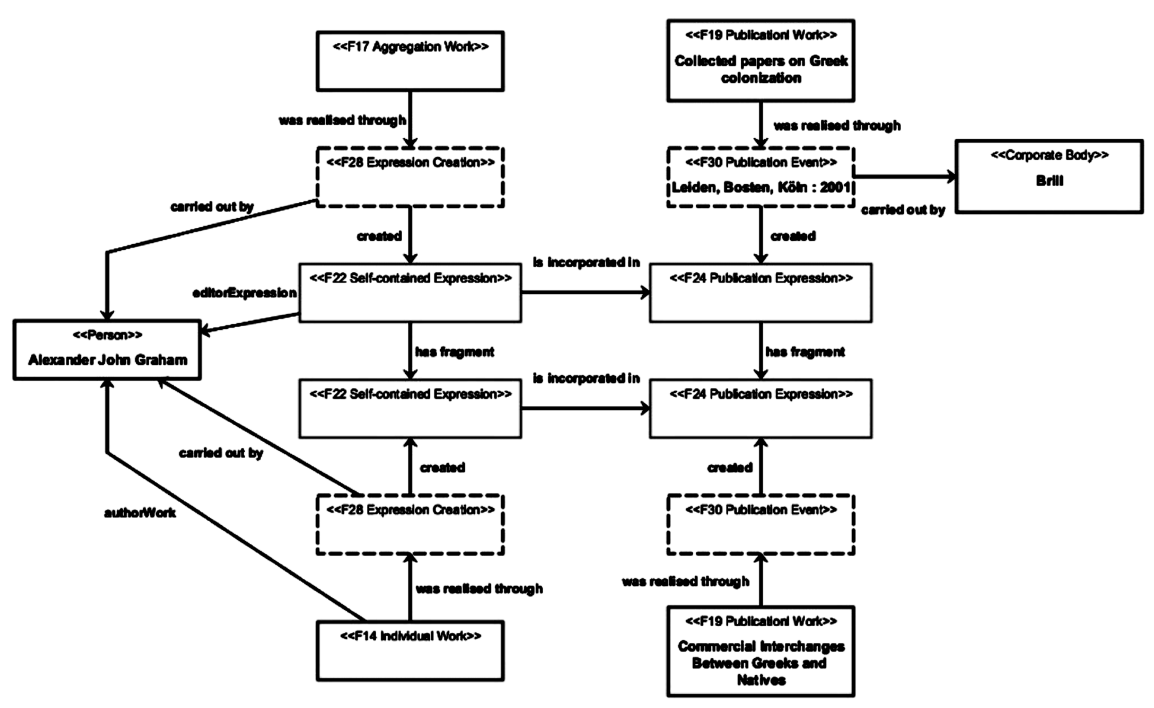

Abbildung 8: Graphische Darstellung von Beispiel 2.

Folgende Präfixe werden verwendet:

eprefix f14: <http://data.archeoinf.tu-dortmund.de/resource/gela/frbroo/F14/> .

@prefix f28_f14: <http://data.archeoinf.tu-dortmund.de/resource/gela/ frbroo/F28/> . 


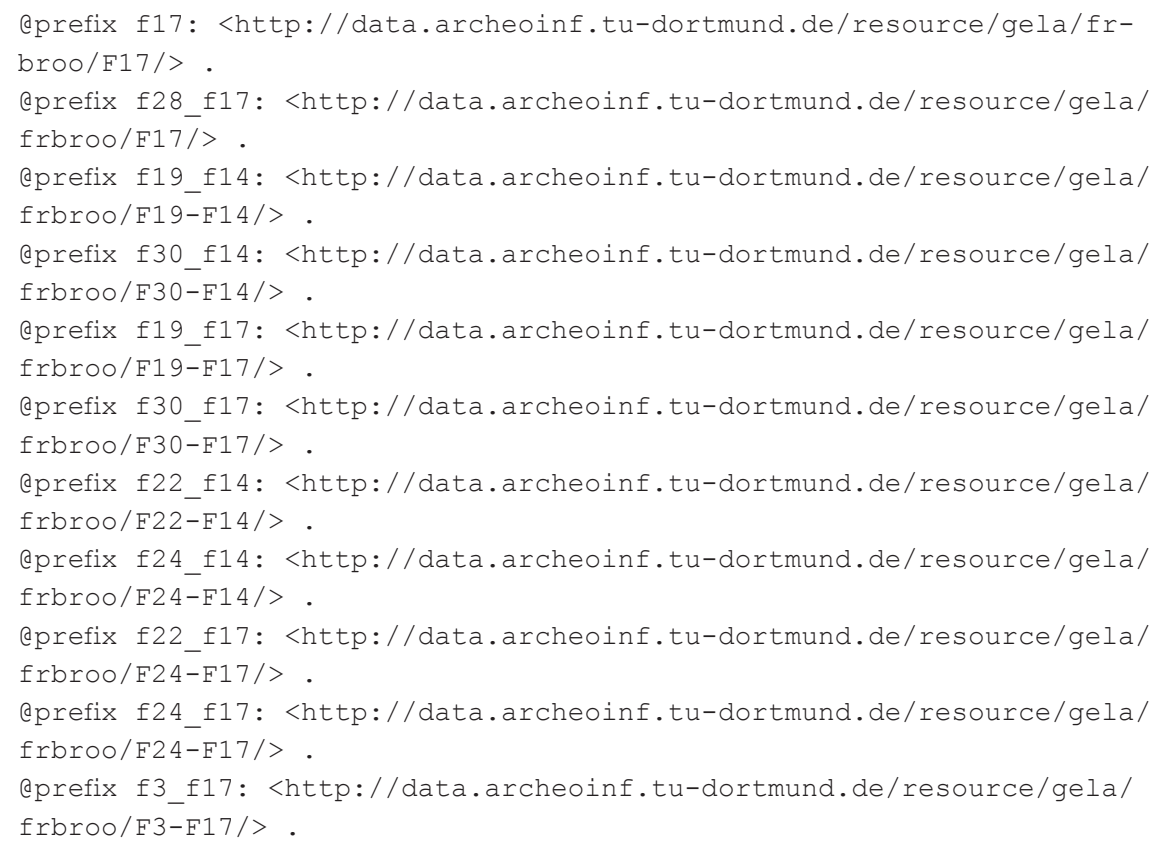

Individual Work des Beitrags:

f14:48473e07-60aa-4858-9a96-133dab42f6f6 efrbroo:R19i_was_realised_through

f28_f14:48473e07-60aa-4858-9a96-133dab42f6f6; rdf:type efrbroo:F14_Individual_Work .

Aggregation Work des Sammelwerks:

f17:48473e07-60aa-4858-9a96-133dab42f6f6

efrbroo:R19i_was_realised_through

f28_f17:48473e07-60aa-4858-9a96-133dab42f6f6 ;

rdf:type efrbroo:F17_Aggregation_Work .

\section{Publication Work des Beitrags:}

f19_f14:48473e07-60aa-4858-9a96-133dab42f6f6

efrbroo:R23i_was_realised_through

f30_f14:48473e07-60aa-4858-9a96-133dab42f6f6 ;

rdaGr1: preferredTitleForTheWork

Commercial Interchanges Between Greeks and Natives ;

rdf:type efrbroo:F19_Publication_Work . 
Publication Work des Sammelwerks:

f19_f17:48473e07-60aa-4858-9a96-133dab42f6f6

efrbroo:R23i_was_realised_through

f30_f17:48473e07-60aa-4858-9a96-133dab42f6f6 ;

rdaGr1: preferredTitleForTheWork

Collected papers on Greek colonization ;

rdf:type efrbroo:F19_Publication_Work .

Self-contained Expression des Beitrags:

f22_f14:48473e07-60aa-4858-9a96-133dab42f6f6

efrbroo:R17i_was_created_by

f28_f14:48473e07-60aa-4858-9a96-133dab42f6f6 ;

efrbroo:R14i_is_incorporated_in

f24_f14:48473e07-60aa-4858-9a96-133dab42f6f6 ;

efrbroo:R15i_is_fragment_of

f22_f17:48473e07-60aa-4858-9a96-133dab42f6f6;

rdaGr1:languageOfTheContentExpression marcLang:eng ;

rdf:type efrbroo:F22_Self-Contained_Expression ;

rdf:type efrbroo:F23_Expression_Fragment .

Publication Expression des Beitrags:

f24_f14:48473e07-60aa-4858-9a96-133dab42f6f6

efrbroo:R24i_was_created_through

f30_f14:48473e07-60aa-4858-9a96-133dab42f6f6 ;

efrbroo:R14_incorporates

f22_f14:48473e07-60aa-4858-9a96-133dab42f6f6 ;

efrbroo:R15i_is_fragment_of

f24_f17:48473e07-60aa-4858-9a96-133dab42f6f6 ;

rdf:type efrbroo:F24_Publication_Expression ;

rdf:type efrbroo:F23_Expression_Fragment .

Self-contained Expression des Sammelwerks:

f22_f17:48473e07-60aa-4858-9a96-133dab42f6f6

efrbroo:R14i_is_incorporated_in

f24_f17:48473e07-60aa-4858-9a96-133dab42f6f6 ;

efrbroo:R17i_was_created_by

f28_f17:48473e07-60aa-4858-9a96-133dab42f6f6 ;

efrbroo:R15_has_fragment

f22_f14:48473e07-60aa-4858-9a96-133dab42f6f6 ;

efrbroo:R4_carriers_provided_by

f3_f17:48473e07-60aa-4858-9a96-133dab42f6f6

rdaGr1:languageOfTheContentExpression marcLang:eng ;

rdf:type efrbroo:F22_Self-Contained_Expression . 
Publication Expression des Sammelwerks:

f24_f17:48473e07-60aa-4858-9a96-133dab42f6f6

efrbroo:R24i_was_created_through

f30_f17:48473e07-60aa-4858-9a96-133dab42f6f6 ;

efrbroo:R14_incorporates

f22_f17:48473e07-60aa-4858-9a96-133dab42f6f6 ;

efrbroo:R15_has_fragment

f24_f14:48473e07-60aa-4858-9a96-133dab42f6f6 ;

rdf:type efrbroo:F24_Publication_Expression .

\section{Mehrbändig begrenzte Werke}

Bei der Abbildung von mehrbändig begrenzten Werken wird es schon etwas komplexer. Es gibt hier zwei zu unterscheidende Fälle: mehrbändiges Werk im Schöpfungsprozess (bibliographische Einheiten) und mehrbändiges Werk im Publikationsprozess (buchbinderische Einheiten).

Bei der Mehrbändigkeit im Schöpfungsprozess findet die Definition der Entität F15 Complex Work Verwendung:

This class comprises works that have more than one work as members. The members of a Complex Work may constitute components of the overall concept or be alternatives to other members of the work. [...] The member relationship of Work is based on the conceptual relationship, and should not be confused with the internal structural parts of an individual expression. ${ }^{24}$

Die Mehrbändigkeit bezieht sich in diesem Fall auf die inhaltliche Ebene. Somit ergibt sich aus dieser Definition direkt die Unterscheidung zur Mehrbändigkeit im Publikationsprozess, bei der sich die Mehrbändigkeit rein praktisch begründet. In letzterem Fall wird die „einbändige“ Expression des Verfassers in mehrere Manifestations aufgeteilt.

Abbildung 9 stellt ein im Publikationsprozess entstehendes mehrbändiges Werk dar, lässt dabei aber den Aspekt des Produktionsprozesses weitestgehend außen vor, da er sich nicht von dem des Kernmodells unterscheidet. Das vom Verfasser initiierte Werk wird im Vorfeld des Publikationsprozesses in mehrere Fragmente zerlegt (F23 Expression Fragment). Diese werden dann jeweils in den Publikationsprozess geleitet und werden somit zu Publication Works. Diese wiederum ergeben gemeinsam ein komplexes Werk im Sinne der FRBRoo-Definition, da hier eine gemeinsame inhaltliche Konzeption - in der Regel durch den Verlag

24 Vgl. ebd. S. 44. 
- zugrunde liegt. Somit sind die den Werken zugrundeliegenden Instanzen der Work Conceptions unterschiedliche Ereignisse. ${ }^{25}$

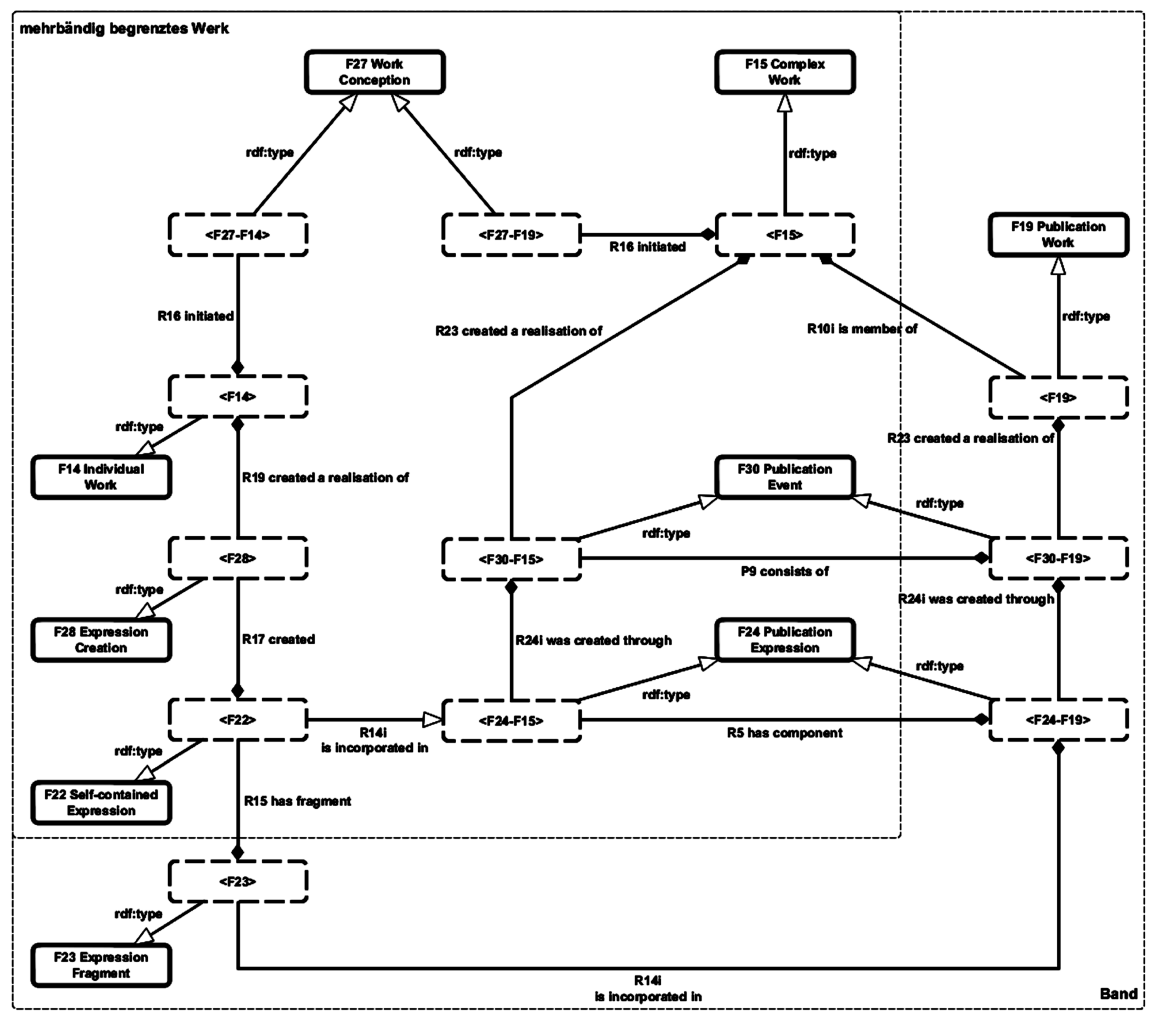

Abbildung 9: Mehrbändig begrenztes Werk im Publikationsprozess.

Die entstandenen Complex Works erhalten jeweils einen eigenen ExpressionZweig. Dieser existiert, sobald das Werk vollständig ist, und da es sich hier um mehrbändig begrenzte Werke handelt, ist bereits von der Vollständigkeit auszugehen. Die Expression Creation-Ereignisse der einzelnen Bände sind somit Teilereignisse der Expression Creation des F15 Complex Work (P9 consists of) und die Expressions der Bände sind Teile der Expression des Complex Work. Bei letzterem ist die Teil-Beziehung nicht mit der Enthaltenseinsbeziehung bei Wiederverwendung einer Expression zu verwechseln. In den FRBRoo heißt es zu R5 has component:

25 Im Abschnitt „Events in FRBROO: How Each Group 1 Entity Comes into Being” stellt Patrick Le Boeuf dar, was genau unter der Work Conception zu verstehen ist. Le Boeuf, Patrick: Strange. 
This property associates an F2 Expression $\mathrm{X}$ with a structural component $\mathrm{Y}$ that conveys in itself the complete concept of a work that is member of (R10) the overall work realized by $\mathrm{X}$. It does not cover the relationship that exists between pre-existing expressions that are re-used in a new, larger expression and that new, larger expression. Such a relationship is modeled by R14 incorporates. ${ }^{26}$

\section{Beispiel 3:}

Grundlagen der praktischen Information und Dokumentation : ein Handbuch zur Einführung in die fachliche Informationsarbeit / Buder, Marianne ; Laisiepen, Klaus.München [u.a.]: Saur, 1990.3., völlig neu gefasste Ausg.

ISBN: 3-598-21253-4

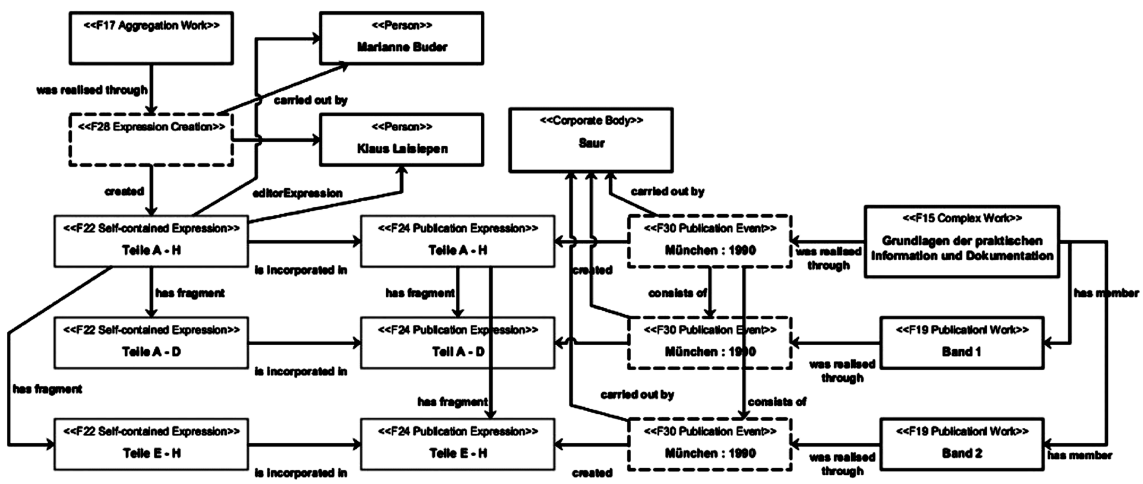

Abbildung 10: Graphische Darstellung von Beispiel 3.

Folgende Präfixe werden verwendet:

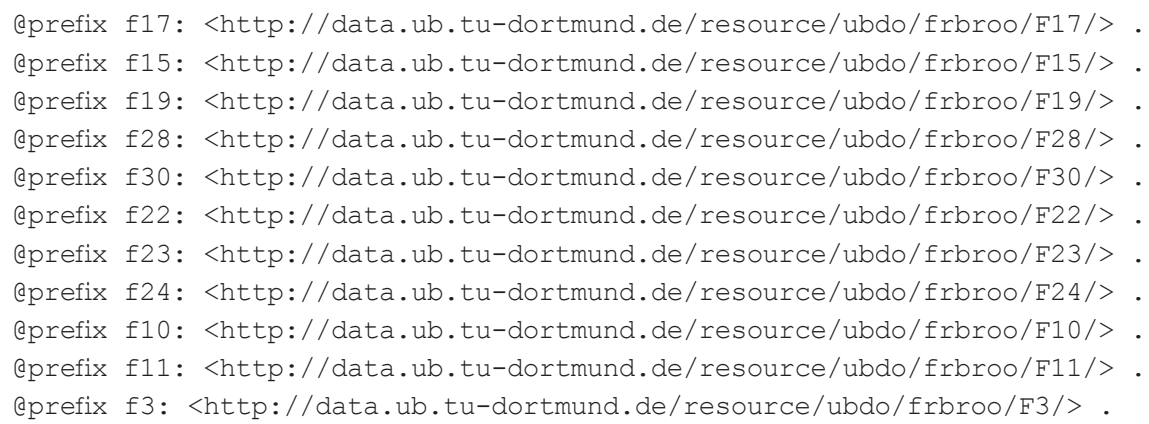

26 Vgl. Bekiari, Chryssoula, Martin Doerr u. Patrick Le Bœuf: Definition. S. 56. 
Aggregation Work:

f17:HT003592036

efrbroo:R19i_was_realised_through f28:HT003592036 ;

rdf:type efrbroo:F17_Aggregation_Work .

Complex Work:

f15:HT003592036

efrbroo:R10_has_member f19:HT003592026 ;

efrbroo:R10_has_member f19:HT003592033 ;

efrbroo:R23i_was_realised_through f30:HT003592036 ;

rdaGr1:titleOfTheWork

Grundlagen der praktischen Information und Dokumentation :

ein Handbuch zur Einführung in die fachliche Informationsarbeit ;

rdf:type efrbroo:F15_Complex_Work .

Publication Works:

f19:HT003592033

efrbroo:R10i_is_member_of f15:HT003592036 ;

efrbroo:R23i_was_realised_through f30:HT003592033 ;

rdaGr1:otherTitleInformation

Grundlagen der praktischen Information und Dokumentation :

ein Handbuch zur Einführung in die fachliche Informationsarbeit / 1 ;

rdf:type efrbroo:F19_Publication_Work .

f19:HT003592026

efrbroo:R10i_is_member_of f15:HT003592036 ;

efrbroo:R23i_was_realised_through f30:HT003592026 ;

rdaGr1:otherTitleInformation

Grundlagen der praktischen Information und Dokumentation :

ein Handbuch zur Einführung in die fachliche Informationsarbeit / 2 ;

rdf:type efrbroo:F19_Publication_Work .

Expression Creation:

f28:HT003592036

ecrm:P14_carried_out_by gnd:105708305 ;

ecrm:P14_carried_out_by f10:HT003592036-1 ;

efrbroo:R17_created f22:HT003592036;

efrbroo:R19_created_a_realisation_of f17:HT003592036 ;

rdf:type efrbroo:F28_Expression_Creation .

Publication Events:

f30:HT003592036

dc:date 1990 ;

dc:place München [u.a.] ;

ecrm:P14F_carried_out_by f11:HT003592033-1 ;

ecrm:P14F_carried_out_by f11:HT003592026-1;

efrbroo:R23_created_a_realisation_of f15:HT003592036 ;

ecrm:P9_consists_of f30:HT003592033 ; 
ecrm:P9_consists_of f30:HT003592026 ;

efrbroo:R24_created f24:HT003592036;

rdf:type efrbroo:F30_Publication_Event .

f30:HT003592033

dc:date 1990 ;

dc:place München [u.a.] ;

ecrm:P14F_carried_out_by f11:HT003592033-1 ;

efrbroo:R23_created_a_realisation_of f19:HT003592033 ;

efrbroo:R24_created f24:HT003592033 ;

ecrm:P9i_forms_part_of f30:HT003592036 ;

rdf:type efrbroo:F30_Publication_Event .

f30:HT003592026

dc:date 1990 ;

dc:place München [u.a.] ;

ecrm:P14F_carried_out_by f11:HT003592026-1 ;

efrbroo:R23_created_a_realisation_of f19:HT003592026 ;

efrbroo:R24_created f24:HT003592026 ;

ecrm:P9i_forms_part_of f30:HT003592036 ;

rdf:type efrbroo:F30_Publication_Event .

Self-contained Expression:

f22:HT003592036

efrbroo:R14i_is_incorporated_in f24:HT003592036 ;

efrbroo:R15_has_fragment $\mathrm{f} 23: \mathrm{HT} 003592026$;

efrbroo:R15_has_fragment f23:HT003592033 ;

efrbroo:R17i_was_created_by f28:HT003592036 ;

rdaGr1:languageOfTheContentExpression marcLang:ger ;

rdaRole:editor gnd:105708305;

rdaRole:editor f10:HT003592036-1 ;

rdf:type efrbroo:F22_Self-Contained_Expression .

Expression Fragments:

f23:HT003592033

efrbroo:R14i_is_incorporated_in f24:HT003592033 ;

efrbroo:R15i_is_fragment_of f22:HT003592036 ;

efrbroo:R4_carriers_provided_by f3:HT003592033 ;

rdaGr1:languageOfTheContentExpression marcLang:ger ;

rdf:type efrbroo:F23_Expression_Fragment .

f23:HT003592026

efrbroo:R14i_is_incorporated_in f24:HT003592026 ;

efrbroo:R15i_is_fragment_of f22:HT003592036 ;

efrbroo:R4_carriers_provided_by f3:HT003592026 ;

rdaGr1:languageOfTheContentExpression marcLang:ger ;

rdf:type efrbroo:F23_Expression_Fragment . 
Publication Expressions:

f24:HT003592036

efrbroo:R14_incorporates f22:HT003592036 ;

efrbroo:R24i_was_created_through f30:HT003592036 ;

efrbroo:R5_has_component f24:HT003592033 ;

efrbroo:R5_has_component f24:HT003592026 ;

rdf:type efrbroo:F24_Publication_Expression .

f24:HT003592033

efrbroo:CLR6i_should_be_carried_by f3:HT003592033 ;

efrbroo:R14_incorporates f23:HT003592033 ;

efrbroo:R24i_was_created_through f30:HT003592033 ;

efrbroo:R5i_is_component_of $\mathrm{f} 24: \mathrm{HT} 003592036$;

rdf:type efrbroo:F24_Publication_Expression .

f24:HT003592026

efrbroo:CLR6i_should_be_carried_by f3:HT003592026 ;

efrbroo:R14_incorporates f23:HT003592026 ;

efrbroo:R24i_was_created_through f30:HT003592026 ;

efrbroo:R5i_is_component_of $\mathrm{f} 24: \mathrm{HT} 003592036$;

rdf:type efrbroo:F24_Publication_Expression .

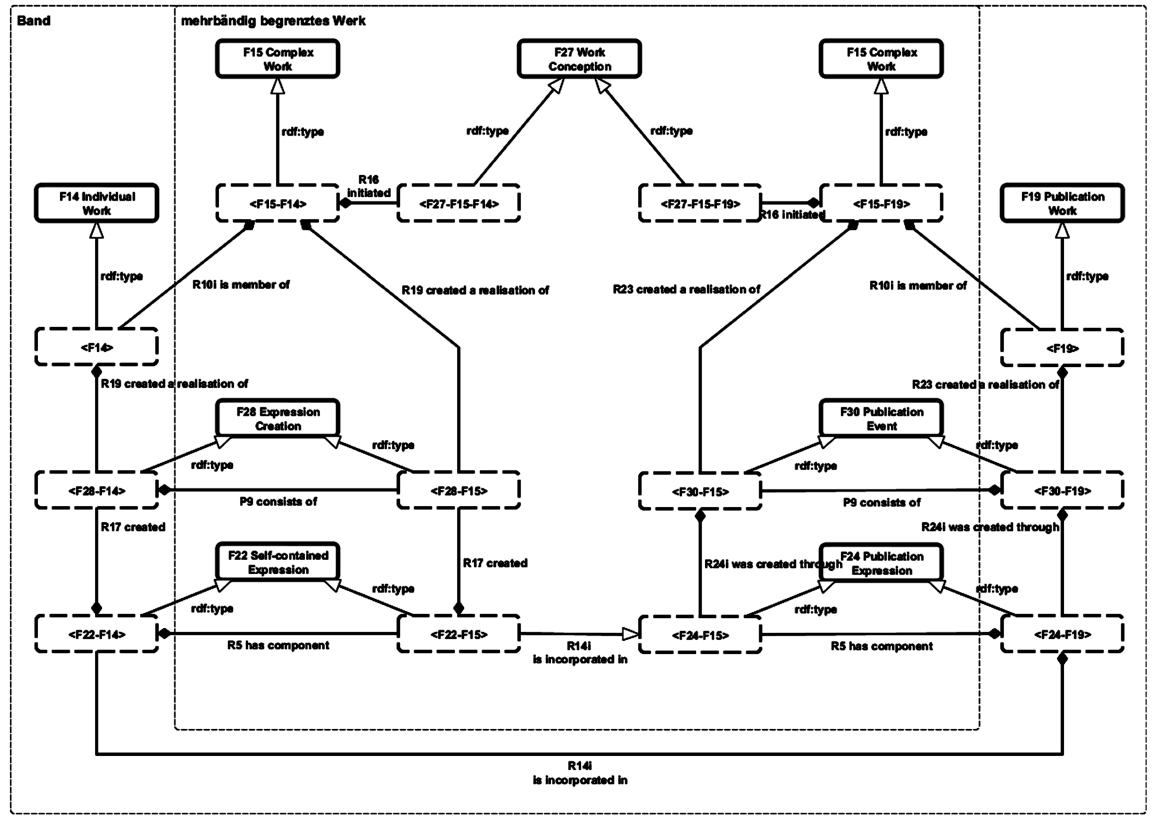

Abbildung 11: Mehrbändig begrenztes Werk im Schöpfungsprozess. 
In Abbildung 11 ist ein im Schöpfungsprozess entstehendes mehrbändiges Werk dargestellt. Im Unterschied zum vorigen Fall findet hier die Zerlegung schon auf Werksebene statt, da der Verfasser schon bei der Konzeption einen Mehrbänder im Sinn hat. Diese Bände werden als Individual Works modelliert und durch jeweils eigene Expressions realisiert. Diese wiederum gehen dann getrennt voneinander in den Publikationsprozess. Wie auch im vorigen Fall, bilden die resultierenden Publication Works eine Einheit in Form eines Complex Work. Für den Fall, dass alle Bände von gleicher Auflage und vom gleichen Verfasser sind, so existiert auch hier eine eigene Realisierung bzw. Expression des Complex Work.

In dem nun folgenden Beispiel gibt es keine gemeinsame Expression des Complex Work. Es zeigt sich hier allerdings die Aufteilung des Schöpfungsprozesses für die einzelnen Bände.

\section{Beispiel 4:}

Analysis / Forster, Otto.

Bd. 1: Differential- und Integralrechnung einer Veränderlichen.

Braunschweig [u.a.]: Vieweg, 2008. 9., überarb. Aufl.

$B d$. 2: Differentialrechnung im R n, gewöhnliche Differentialgleichungen.

Braunschweig [u.a.]: Vieweg, 2008. 8., aktualisierte Aufl.

$B d$. 3: Integralrechnung im $R$ n mit Anwendungen.

Braunschweig [u.a.]: Vieweg, 2009. 5., aktualisierte Aufl. 


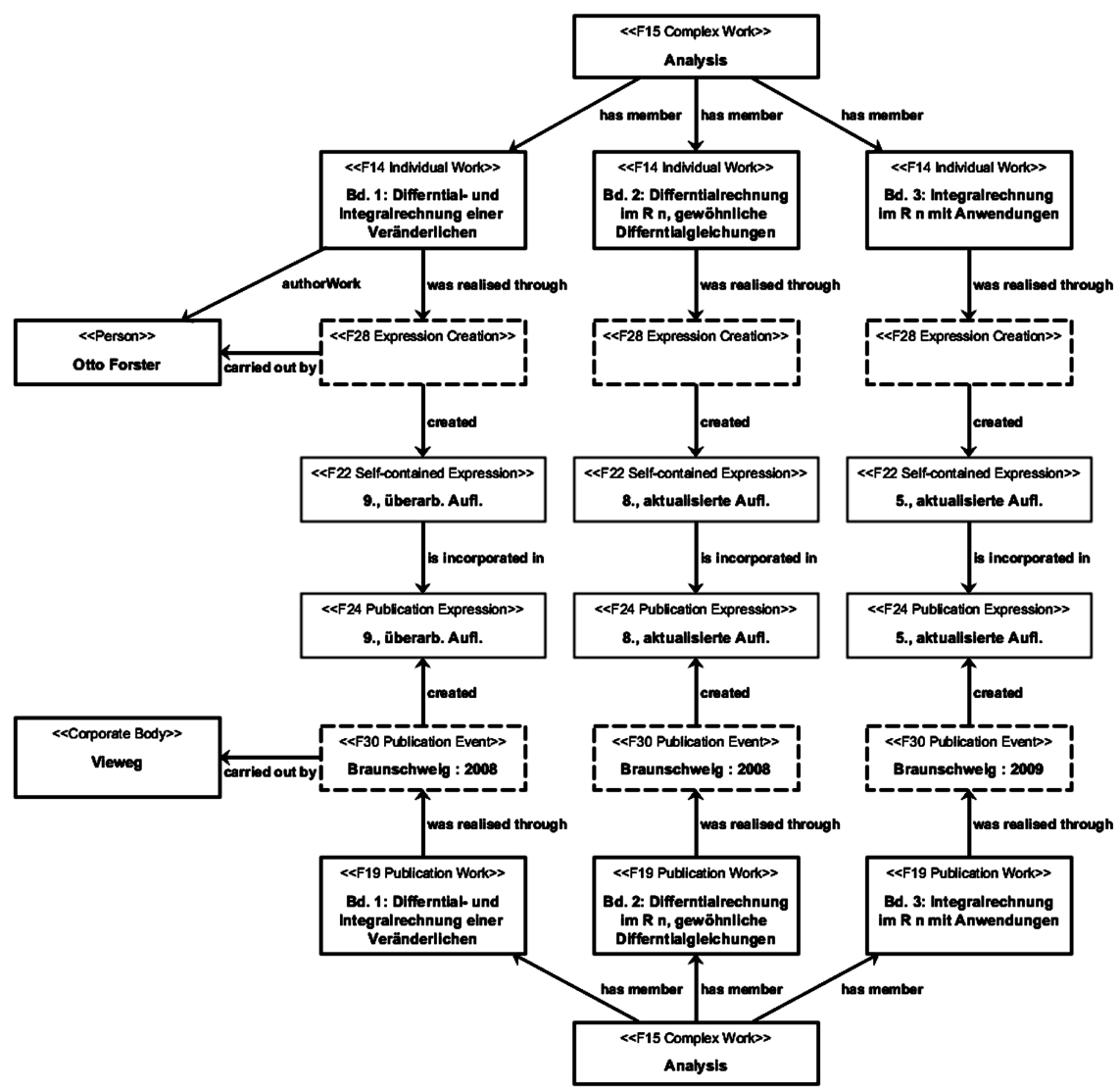

Abbildung 12: Graphische Darstellung von Beispiel 4.

Folgende Präfixe werden verwendet:

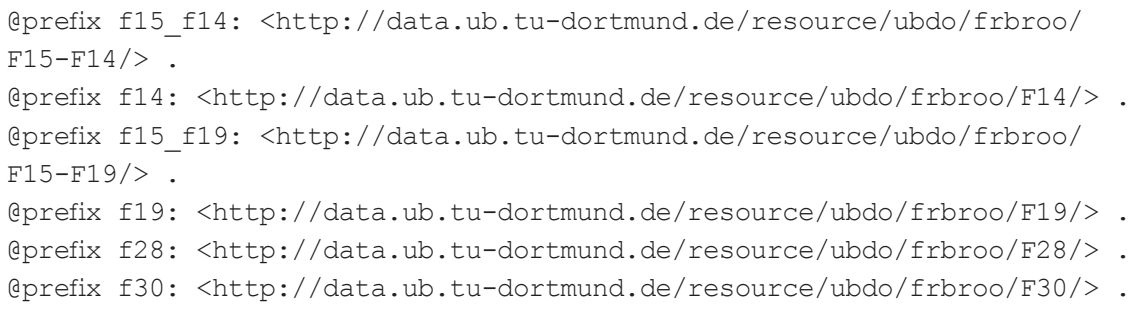


Complex Works:

f15_f14:HT001321758

efrbroo:R10_has_member f14:HT015417495 ;

efrbroo:R10_has_member f14:HT015618061 ;

efrbroo:R10_has_member f14:HT015770825 ;

rdf:type efrbroo:F15_Complex_Work .

f15_f19:HT001321758

efrbroo:R10_has_member f19:HT015417495 ;

efrbroo:R10_has_member f19:HT015618061 ;

efrbroo:R10_has_member f19:HT015770825 ;

rdaGr1:titleOfTheWork Analysis ;

rdf:type f15:F15_Complex_Work .

Individual Works:

f14:HT015417495

efrbroo:R10i_is_member_of f15_f14:HT001321758 ;

efrbroo:R19i_was_realised_through f28:HT015417495 ;

rdaRole:authorWork gnd:115448942 ;

rdf:type efrbroo:F14_Individual_Work .

f14:HT015618061

efrbroo:R10i_is_member_of f15_f14:HT001321758 ;

efrbroo:R19i_was_realised_through f28:HT015618061 ;

rdaRole:authorWork gnd:115448942;

rdf:type efrbroo:F14_Individual_Work

f14:HT015770825

efrbroo:R10i_is_member_of f15_f14:HT001321758 ;

efrbroo:R19i_was_realised_through f28:HT015770825 ;

rdaRole:authorWork gnd:115448942;

rdf:type efrbroo:F14_Individual_Work .

Publication Works:

f19:HT015417495

efrbroo:R10i_is_member_of f15_f19:HT001321758 ;

efrbroo:R23i_was_realised_through f30:HT015417495 ;

rdaGr1:otherTitleInformation Analysis / 1 ;

rdaGr1:titleOfTheWork

Differential- und Integralrechnung einer Veränderlichen ;

rdf:type efrbroo:F19_Publication_Work .

f19:HT015618061

efrbroo:R10i_is_member_of f15_f19:HT001321758 ;

efrbroo:R23i_was_realised_through f30:HT015618061 ;

rdaGr1:otherTitleInformation Analysis / 2 ;

rdaGr1:titleOfTheWork

Differentialrechnung im R n, gewöhnliche Differentialgleichungen ;

rdf:type efrbroo:F19_Publication_Work .

f19:HT015770825 


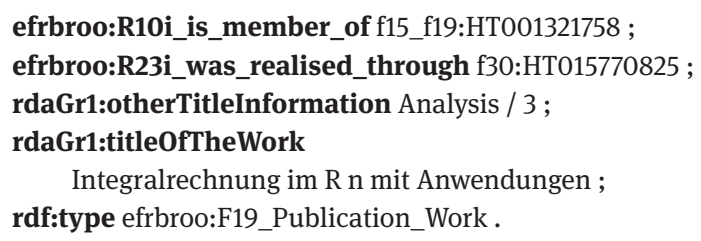

Die weiteren Entitäten sind nun analog zum vorigen Beispiel.

\section{Fortlaufende Sammelwerke}

Bei der Definition der FRBRoo wurde darauf geachtet, dass die Begrifflichkeiten der FRBR noch präzisiert werden. Diese begriffliche Abklärung ergibt sich in vielen Fällen aus der Darstellung der bibliographischen Information als Prozess. Als ein Beispiel wurde oben schon die Klasse Work mit ihren präzisierenden Unterklassen dargestellt. Insbesondere wurde mit der Klasse F18 Serial Work eine Möglichkeit geschaffen, die Unklarheiten zu Serials, also den fortlaufenden Sammelwerken, in den FRBR zu beseitigen. Dort ist klar beschrieben, dass eine Manifestation in dem Sinn abgeschlossen sein muss, dass man ein Exemplar kaufen und ins Regal stellen kann. Bei fortlaufenden Sammelwerken ist das nicht möglich, solange es weitere Bände - beispielsweise in Form von Jahrgängen und Heften - gibt. In FRBRoo heißt es dazu:

\footnotetext{
This class comprises works that are, or have been, planned to result in sequences of manifestations with common features. Whereas a work can acquire new members over the time it evolves Expressions and Manifestations are identified with a certain state achieved at a particular point in time. Therefore there is in general no single expression or manifestation representing a complete serial work, unless the serial work is ended. [...] Serial Works may or may not have a plan for an overall expression. ${ }^{27}$
}

Es ist $\mathrm{zu}$ beachten, dass FRBRoo keine Unterscheidung zwischen Serien und Reihen bzw. Periodika macht, da der Unterschied „nur“ in der Art der Erscheinungsweise liegt und dies über andere Mechanismen modellierbar ist. Somit ergeben sich zwei Fälle: fortlaufende Sammelwerke mit eingebetteten mehrbändigen Werken und fortlaufende Sammelwerke ohne eingebettete mehrbändige Werke. Zu Ersteren zählen beispielsweise Zeitschriften mit Jahrgangzählung.

Fortlaufende Sammelwerke mit eingebetteten mehrbändigen Werken

27 Vgl. ebd. S. 46. 
Nach dem, was in diesem Beitrag bisher gezeigt wurde, lässt sich ein mehrbändiges Werk innerhalb eines fortlaufenden Sammelwerks als F15 Complex Work beschreiben. Dass dies auch im Falle von Periodika gilt, in dem die Jahrgänge in der Regel Fortsetzungswerke sind, wird auch durch folgendes Zitat aus der Definition zu F15 Complex Work deutlich: „One part may not be finished when another is already revised."28

Die Komponente des unbestimmten Endes eines fortlaufenden Sammelwerks entsteht also bei der Einbindung der Complex Works der Mehrbänder in das Serial Work. In Abbildung 11 wird das F15 Complex Work zum Publikationsprozess dann noch zusätzlich um die Struktur des F18 Serial Work erweitert.

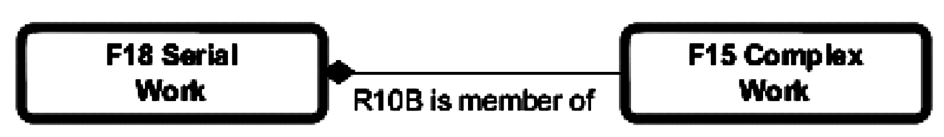

\section{Beispiel 5:}

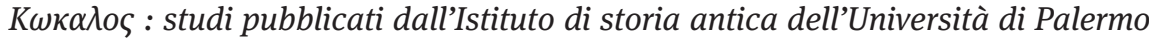
(Kokalos). Volume 45. Heft ohne Angabe.

Folgende Präfixe werden verwendet:

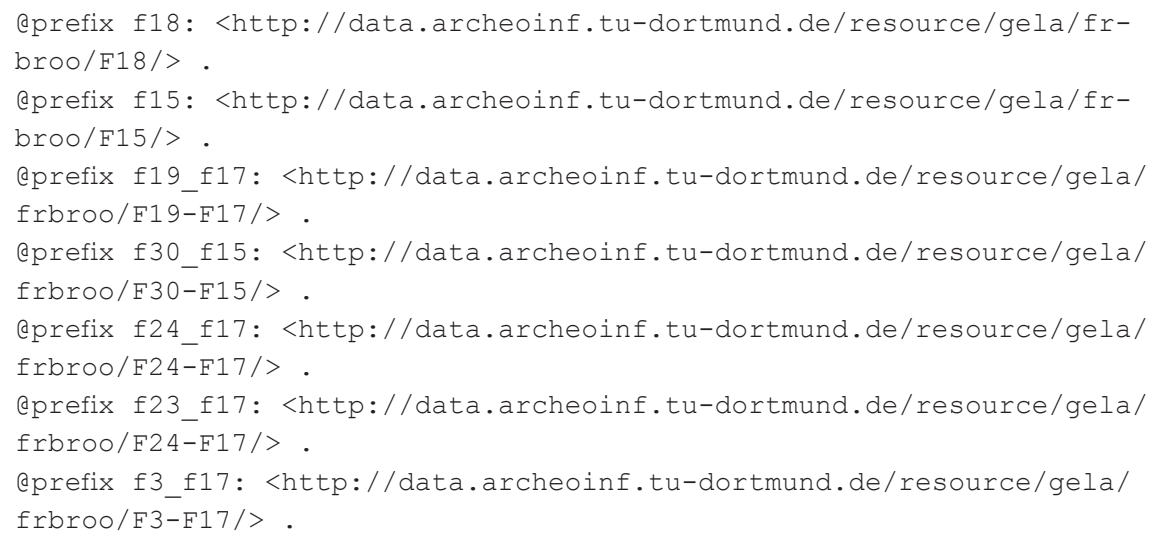

28 Vgl. ebd. S. 44. 
Serial Work (Zeitschrift):

f18:d53bedeb-7b56-4f7e-9d1f-d869e7910184

efrbroo:R10_has_member

f15:d53bedeb-7b56-4f7e-9d1f-d869e7910184 ;

rdaGr1:preferredTitleForTheWork

K $\omega \kappa \alpha \lambda$ oৎ : studi pubblicati dall'Istituto di storia antica dell'Università di Palermo ;

rdaGr1:abbreviatedTitle Kokalos ;

rdf:type efrbroo:F18_Serial_Work ;

Complex Work (Jahrgang, Volume):

f15:d53bedeb-7b56-4f7e-9d1f-d869e7910184

efrbroo:R10i_is_member_of

f18:d53bedeb-7b56-4f7e-9d1f-d869e7910184 ;

efrbroo:R10_has_member

f19_f17:d53bedeb-7b56-4f7e-9d1f-d869e7910184 ;

efrbroo:R23i_was_realised_through

f30_f15:d53bedeb-7b56-4f7e-9d1f-d869e7910184 ;

rdaGr1:titleOfTheWork

K $\omega \kappa \alpha \lambda$ o : studi pubblicati dall'Istituto di storia antica dell'Università di Palermo / 45 ;

rdf:type efrbroo:F15_Complex_Work .

Publication Work (Heft):

f19_f17:d53bedeb-7b56-4f7e-9d1f-d869e7910184

efrbroo:R10i_is_member_of

f15:d53bedeb-7b56-4f7e-9d1f-d869e7910184 ;

efrbroo:R23i_was_realised_through

f30_f17:d53bedeb-7b56-4f7e-9d1f-d869e7910184 ;

rdaGr1:preferredTitleForTheWork

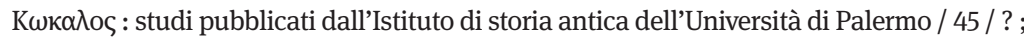

rdf:type efrbroo:F19_Publication_Work .

Manifestation Product Type (Manifestation des Heftes):

f3_f17:d53bedeb-7b56-4f7e-9d1f-d869e7910184

efrbroo:CLR6_should_carry

f24_f17:d53bedeb-7b56-4f7e-9d1f-d869e7910184 ;

efrbroo:R4i_comprises_carriers_of

f23_f17:d53bedeb-7b56-4f7e-9d1f-d869e7910184;

rdaGr1:identifierForTheManifestation [ISSN] 0454-1596 ;

rdaGr1:identifierForTheManifestation [ISSN] 0392-0887 ;

rdf:type efrbroo:F3_Manifestation_Product_Type ;

rdf:type frbr:Manifestation ;

Fortlaufende Sammelwerke ohne eingebettete mehrbändige Werke

Dieser Fall lässt sich in ähnlicher Weise aus den vorher gezeigten Modellen herleiten. Da es sich hier um Einzelwerke und Sammelwerke handelt, die in einer 
Reihe erschienen sind, werden die Individual Works und somit auch die Aggregation Works als member eines Serial Works definiert.

\section{Beispiel 6:}

Handbuch Bibliothek 2.0 / Bergmann, Julia [Hrsg.] ; Danowski, Patrick [Hrsg.]. In: Bibliothekspraxis.München: De Gruyter Saur, 2010.

Folgende Präfixe werden verwendet:

@prefix f18: <http://data.ub.tu-dortmund.de/resource/ubdo/frbroo/F18/>.
aprefix f19: <http://data.ub.tu-dortmund.de/resource/ubdo/frbroo/F19/>.
aprefix f30: <http://data.ub.tu-dortmund.de/resource/ubdo/frbroo/F30/>.

Serial Work:

f18:HT016530358

efrbroo:R10_has_member f19:HT016530358 ;

rdaGr1:preferredTitleForTheWork Bibliothekspraxis ;

rdf:type efrbroo:F18_Serial_Work .

Publication Work:

f19:HT016530358

efrbroo:R10i_is_member_of f18:HT016530358 ;

efrbroo:R23i_was_realised_through f30:HT016530358 ;

rdaGr1:preferredTitleForTheWork Handbuch Bibliothek 2.0 ;

rdf:type efrbroo:F19_Publication_Work .

\section{Fazit/Ausblick}

Im vorliegenden Beitrag wurde gezeigt, dass die viel diskutierten Probleme mit den fortlaufenden Sammelwerken zumindest in der Darstellung für die Linked Data Umgebung lösbar sind. Trotzdem ist die beschriebene Lösung noch nicht perfekt. Zwar erfolgte die Abbildung der Attribute weitestgehend nach $R D A$ und somit auch auf die FRBRoo-Entitäten, jedoch sind hier noch nicht alle Attribute und somit bibliographische Informationen auf die Entitäten verteilt. Auch hat in den bisherigen Implementierungen noch keine „richtige“ FRBRisierung stattgefunden, d.h., dass es für viele FRBR-Entitäten noch mehrere URIs gibt, da jeweils ein (MODS-)Datensatz auf alle beschriebenen Entitäten abgebildet wurde. ${ }^{29}$

29 Da die derzeit betriebenen Katalogsysteme nur Manifestationen und Exemplare abbilden, 
Um diese FRBRisierung bald in Angriff zu nehmen, wird die Bundle-Ontologie $^{30}$ Verwendung finden. Diese ermöglicht es, Objekte zusammenzufassen und gleichzeitig zu dokumentieren, auf welcher Grundlage die Bündelung stattgefunden hat.

Für das hier beschriebene Verfahren der Umwandlung der bibliographischen Datensätze in FRBRoo-Entitäten und -Relationen gibt es derzeit zwei Anwendungsbeispiele. Zum einen ist dies das Linked-Open-Library-Data-Portal der Universitätsbibliothek Dortmund und zum anderen das DFG-Projekt ArcheoInf, welches - basierend auf dem hier beschriebenen Ansatz - archäologische, mittels des CIDOC CRM erfasste Forschungsdaten mit bibliographischen Daten verbindet. Näheres zum Projekt ArcheoInf findet sich im Beitrag „Open Data und Linked Data in einem Informationssystem für die Archäologie“ in diesem Band.

\section{Quellen}

ArcheoInf Dokumentenrepositorium. http://archeoinf.ub.rub.de/ (1.7.2012).

DCMI Metadata Terms. http://purl.org/dc/elements/1.1 / (1.7.2012).

Koreferenzen im Semantic Web. https://wiki1.hbz-nrw.de/display/SEM/Koreferenzen+im+Sem antic+Web\#KoreferenzenimSemanticWeb-bundle (1.7.2012).

Universitätsbibliothek Dortmund: Offene bibliographische Daten an der Universitätsbibliothek Dortmund. http://data.ub.tu-dortmund.de/projekte/offene-daten/ (11.5.2012).

The "xml:" Namespace. http://www.w3.org/XML/1998/namespace (1.7.2012).

The OWL 2 Schema vocabulary (OWL 2). http://www.w3.org/2002/07/owl\# (1.7.2012).

The RDF vocabulary. http://www.w3.org/1999/02/22-rdf-syntax-ns\# (1.7.2012).

SKOS Simple Knowledge Organization System Namespace Document - HTML Variant, 18 August 2009 Recommendation Edition. http://www.w3.org/2004/02/skos/core\# (1.7.2012).

Erlangen-CRM. http://erlangen-crm.org/120111/ (1.7.2012).

Bundle Ontology. http://benosteen.com/bundle.rdf (1.7.2012).

Becker, Hans-Georg u. Förster, Frank: Vernetztes Wissen - Ereignisse in der bibliografischen Dokumentation. In: Zeitschrift für Bibliothekswesen und Bibliographie 57 (2010) H. 1. S. 15-25.

Becker, Hans-Georg: MODS2FRBROO. Ein Tool zur Anbindung von bibliografischen Daten an eine Ontologie für Begriffe und Informationen im Bereich des kulturellen Erbes. In: Semantic Web \& Linked Data. Elemente zukünftiger Informationsinfrastrukturen ; 1. DGI-Konferenz ; 62. Jahrestagung der DGI ; Frankfurt am Main, 7. - 9. Oktober 2010 ; Proceedings. Hrsg. von Marlies Ockenfeld. Frankfurt am Main: DGI Dt. Ges. für Informati-

sind für die zugehörigen Werke und Expressionen in der Regel keine eindeutigen Identifikatoren vorhanden. Die bisherigen auf dem hier beschriebenen Modell basierenden Implementierungen prägen durch die Nutzung der IDs der Manifestationen somit teilweise mehrere URIs für identische Expressionen und Werke.

30 Bundle Ontology: Bundle. 
onswiss. und Informationspraxis 2010. http://hdl.handle.net/2003/27402 (24.06.2012) S. 77-86.

Bekiari, Chryssoula, Martin Doerr u. Patrick Le Bœuf: Definition of Object-Oriented FRBR. http:// www.cidoc-crm.org/docs/frbr_0o/frbr_docs/FRBRoo_V1.0.2.pdf (1.7.2012).

Heath, Tom u. Christian Bizer: Linked Data. Evolving the web into a global data space. 1. Aufl. San Rafael, Calif: Morgan\& Claypool 2011 (=Synthesis lectures on the semantic web theory and technology 1). http://linkeddatabook.com/editions/1.0 / (24.06.2012)

Hohmann, Georg: Die Anwendung des CIDOC CRM für die semantische Wissensrepräsentation in den Kulturwissenschaften. In: Wissensspeicher in digitalen Räumen. Nachhaltigkeit, Verfügbarkeit, semantische Interoperabilität ; Proceedings der 11. Tagung der Deutschen Sektion der Internationalen Gesellschaft für Wissensorganisation, Konstanz, 20. bis 22. Februar 2008. Hrsg. von Jörn Sieglerschmidt. Würzburg: Ergon-Verl 2010. S. 211-222.

ICOM: CIDOC CRM Mappings, Specializations and Data Examples. http://www.cidoc-crm.org/ crm_mappings.html (1.7.2012).

Le Boeuf, Patrick: A Strange Model Named FRBROO. In: Cataloging \& Classification Quarterly: Routledge 2012. http://dx.doi.10.1080/01639374.2012.679222 (24.6.2012).

Mazurek, Cezary, Krysztof Sielski u. a.: From MARC21 and Dublin Core, through CIDOC CRM. First Tenuous Steps towards Representing Library Data in FRBRoo. Accepted Paper for the CIDOC Conference 2012, Helsinki. http://www.cidoc2012.fi/en/File/1611/mazurek.pdf (1.7.2012).

Pohl, Adrian: Serials and FRBR. https://wiki1.hbz-nrw.de/display/SEM/2011/10/17/ Serials+and+FRBR (1.7.2012).

Riva, Pat, Martin Doerr u. Maja Žumer: FRBRoo: eine Basis zur gemeinsamen Informationsdarstellung in Kulturerbe-Institutionen. http://ifla.queenslibrary.org/IV/ifla74/ papers/156-Riva_Doerr_Zumer-trans-de.pdf (1.7.2012).

The Registry!: FRBR Entities for RDA. http://rdvocab.info/uri/schema/FRBRentitiesRDA/ (1.7.2012).

The Registry!: RDA Group 1 Elements. http://RDVocab.info/Elements/ (1.7.2012). The Registry!: RDA Group 2 Elements. http://RDVocab.info/ElementsGr2/ (1.7.2012). The Registry!: RDA Group 3 Elements. http://RDVocab.info/ElementsGr3/ (1.7.2012). The Registry!: RDA Roles. http://RDVocab.info/roles/ (1.7.2012).

The Registry!: The RDA (Resource Description and Access) Vocabularies. http://rdvocab.info/ (1.7.2012). 